\title{
Discrete Tomography through Distribution Theory
}

\author{
By
}

Fumio HAZAMA*

\begin{abstract}
Discrete tomography concerns with the problem of reconstruction of a function $f$ on $\mathbf{Z}^{n}$ from various sums $f_{\mathbf{t}+\mathbf{v}}=\sum_{\mathbf{x} \in \mathbf{t}+\mathbf{v}} f(\mathbf{x}), \mathbf{v} \in \mathbf{Z}^{n}$, where $\mathbf{t}$ is a fixed finite subset of $\mathbf{Z}^{n}$. In this paper we focus on the structure of the set of functions satisfying $f_{\mathbf{t}+\mathbf{v}}=$ 0 for any $\mathbf{v}$. Through the theory of distributions we deduce a dimension formula for the set of solutions. An intimate connection between the problem and certain types of PDE is revealed too, and it enables one to obtain an efficient algorithm, which constructs a solution from the corresponding PDE.
\end{abstract}

\section{$\S 1 . \quad$ Introduction}

Let $\mathbf{A}=(\mathbf{C})^{\mathbf{Z}^{n}}$ denote the set of $\mathbf{C}$-valued functions on $\mathbf{Z}^{n}$. An element of $\mathbf{A}$ is called an array. An array with finite support is called a window and let $\mathbf{W}$ denote the set of windows. For any $(\mathbf{a}, \mathbf{t}) \in \mathbf{A} \times \mathbf{W}$, let $d_{\mathbf{t}}(\mathbf{a})=\sum_{\mathbf{i} \in \mathbf{Z}^{n}} \mathbf{t}_{\mathbf{i}} \mathbf{a}_{\mathbf{i}}$. Discrete tomography concerns with the problem of reconstruction of an array $\mathbf{a}$ from the set of values $d_{\mathbf{t}+\mathbf{p}}(\mathbf{a}), \mathbf{p} \in \mathbf{Z}^{n}$, for a fixed window $\mathbf{t} \in \mathbf{W}$. Therefore it is of fundamental importance to determine the structure of the set of a such that $d_{\mathbf{t}+\mathbf{p}}(\mathbf{a})=0$ for any $\mathbf{p} \in \mathbf{Z}^{n}$. Such an array $\mathbf{a}$ is said to be a zero-sum array for $\mathbf{t}$. In the previous papers [3, 4], we deal with bounded zero-sum arrays and derive a dimension formula for the space of bounded zero-sum arrays from the investigation of the annihilator of the Dirac delta function. The purpose of the present paper is to extend the scope and to investigate zero-sum arrays of polynomial growth. As in the previous papers, the theory of distributions plays

\footnotetext{
Communicated by T. Kawai. Received May 31, 2007. Revised November 15, 2007.

2000 Mathematics Subject Classification(s): Primary 39A12; Secondary 13N10, 35G05.

*Department of Natural Sciences, College of Science and Engineering, Tokyo Denki University, Hatoyama, Saitama, 350-0394, Japan.
} 
an essential role for our investigation. For our purpose, however, we realize that we need to investigate not only the annihilator of the Dirac delta function but also that of its higher derivatives. Thus we are led naturally to the study of our problem in the light of Weyl algebra and its representation. As a result we find that we can associate a partial differential operator $D_{\mathbf{t}}$ with a window $\mathbf{t}$, and there is a natural one-to-one correspondence between the space of zerosum arrays for $\mathbf{t}$ and that of polynomial solutions for $D_{\mathbf{t}}$. Furthermore we find an inductive procedure for construction of polynomial solutions for PDE which arises in this way. Thus we obtain an efficient algorithm to determine the structure of the space of zero-sum arrays.

The plan of the paper is as follows. In Section two we formulate the problem of our main concern. Recalling some results obtained in $[3,4]$, we see that a crucial role is played by the annihilator of the Dirac delta function and its higher derivatives. Section three deals with the structure of the annihilator, and establishes a result (Theorem 3.2), which provides us with a natural oneto-one correspondence between the space of zero-sum arrays for a window and the space of polynomial solutions of the associated partial differential equation. The next two sections are concerned with the structure of the space of polynomial solutions of PDE arising in this way. Section four provides us with a dimension formula (Theorem 4.3) for polynomial solutions. Through the oneto-one correspondence, this in turn gives us a dimension formula for the space of zero-sum arrays (Theorem 4.4). It follows from the formula that the generating function for the dimensions is always a rational function of quite a simple form (Proposition 4.6). Section five establishes an inductive procedure (Proposition 5.4) for construction of a solution from that for PDE in lesser number of variables. In section six we examine the efficiency of our algorithm through several examples of windows.

The author would like to thank the referee for his/her careful reading of the manuscript and valuable suggestions.

\section{$\S 2 . \quad$ Problem Setting}

In this section we fix some notation and describe the main problem of our concern in this paper.

Let $\mathbf{A}=(\mathbf{C})^{\mathbf{Z}^{n}}$ denote the set of $\mathbf{C}$-valued functions on $\mathbf{Z}^{n}$. We write its element in the form $\mathbf{a}=\left(\mathbf{a}_{\mathbf{i}}\right)$ where $\mathbf{i}=\left(i_{1}, \cdots, i_{n}\right) \in \mathbf{Z}^{n}$ and $\mathbf{a}_{\mathbf{i}} \in \mathbf{C}$. We call an element of $\mathbf{A}$ an array. For any array $\mathbf{a}=\left(\mathbf{a}_{\mathbf{i}}\right)$, let $\operatorname{supp} \mathbf{a}=\left\{\mathbf{i} \in \mathbf{Z}^{n} ; \mathbf{a}_{\mathbf{i}} \neq\right.$ $0\} \subset \mathbf{Z}^{n}$ and call it the support of $\mathbf{a}$. An array with finite support is said to be a window, and the set of windows is denoted by $\mathbf{W}$. For any window $\mathbf{t}=\left(\mathbf{t}_{\mathbf{i}}\right)$ and 
for any array $\mathbf{a}=\left(\mathbf{a}_{\mathbf{i}}\right)$, let $d_{\mathbf{t}}(\mathbf{a})=\sum_{\mathbf{i} \in \mathbf{Z}^{n}} \mathbf{t}_{\mathbf{i}} \mathbf{a}_{\mathbf{i}}$. For any $\mathbf{p} \in \mathbf{Z}^{n}$, the translated window $\mathbf{t}+\mathbf{p}$ is defined by the rule $(\mathbf{t}+\mathbf{p})_{\mathbf{i}}=\mathbf{t}_{\mathbf{i}-\mathbf{p}}, \mathbf{i} \in \mathbf{Z}^{n}$. The object of our main concern in this paper is the space

$$
\mathbf{A}_{\mathbf{t}}=\left\{\mathbf{a} \in \mathbf{A} ; d_{\mathbf{t}+\mathbf{p}}(\mathbf{a})=0 \quad \text { for any } \quad \mathbf{p} \in \mathbf{Z}^{n}\right\}
$$

which we call the space of zero-sum arrays for $\mathbf{t}$. In the previous papers [3, 4], we investigate the subspace of $\mathbf{A}_{\mathbf{t}}$ consisting of bounded zero-sum arrays. In this paper, we are interested in the zero-sum arrays of polynomial growth. More precisely, we introduce the set $\mathbf{A}^{N}=\left\{\mathbf{a} \in \mathbf{A} ; \mathbf{a}_{\mathbf{k}}=O\left(|\mathbf{k}|^{N}\right)\right.$ as $\left.|\mathbf{k}| \rightarrow \infty\right\}$, and put $\mathbf{A}^{\text {poly }}=\bigcup_{N>0} \mathbf{A}^{N}$. What we want to understand in this paper is the structure of $\mathbf{A}_{\mathbf{t}}^{\text {poly }}=\mathbf{A}_{\mathbf{t}} \cap \mathbf{A}^{\text {poly }}$ as well as $\mathbf{A}_{\mathbf{t}}^{N}=\mathbf{A}_{\mathbf{t}} \cap \mathbf{A}^{N}$. As in [3], we will see that a crucial role for our investigation is played by the characteristic polynomial of $\mathbf{t}=\left(\mathbf{t}_{\mathbf{i}}\right) \in \mathbf{W}$, defined by $m_{\mathbf{t}}(\mathbf{z})=\sum_{\mathbf{i} \in \mathbf{Z}^{n}} \mathbf{t}_{\mathbf{i}} \mathbf{z}^{\mathbf{i}} \in \mathbf{C}\left[z_{1}, z_{1}^{-1}, \cdots, z_{n}, z_{n}^{-1}\right]$, where $\mathbf{z}=\left(z_{1}, \cdots, z_{n}\right)$ and $\mathbf{z}^{\mathbf{i}}=z_{1}^{i_{1}} \cdots z_{n}^{i_{n}}$. Let $\mathbf{T}=\{z \in \mathbf{C} ;|z|=1\}$ and let $\iota: \mathbf{T}^{n} \rightarrow \mathbf{T}^{n}$ denote the automorphism of the $n$-torus defined by $\iota\left(z_{1}, \cdots, z_{n}\right)=$ $\left(z_{1}^{-1}, \cdots, z_{n}^{-1}\right)$. We put $m_{\mathbf{t}}^{*}=\iota^{*}\left(m_{\mathbf{t}}\right)$ so that $m_{\mathbf{t}}^{*}(\mathbf{z})=m_{\mathbf{t}}(\iota(\mathbf{z}))$. For any subset $X \subset \mathbf{T}^{n}$, we denote the zero locus $\left\{\mathbf{z} \in X ; m_{\mathbf{t}}(\mathbf{z})=0\right\}$ by $V_{X}\left(m_{\mathbf{t}}\right)$. In [3], we find that the structure of $\mathbf{A}_{\mathbf{t}}^{0}$, the space of bounded zero-sum arrays, is controlled by the zero locus $V_{\mathbf{T}^{n}}\left(m_{\mathbf{t}}^{*}\right)$. Among other things we have proved there that $\operatorname{dim}_{\mathbf{C}} \mathbf{A}_{\mathbf{t}}^{0}=\#\left(V_{\mathbf{T}^{n}}\left(m_{\mathbf{t}}^{*}\right)\right)$. In the present paper, we will show that the structure of $\mathbf{A}_{\mathbf{t}}^{\text {poly }}\left(\supset \mathbf{A}_{\mathbf{t}}^{0}\right)$ is controlled not only by $V_{\mathbf{T}^{n}}\left(m_{\mathbf{t}}^{*}\right)$ but also a certain partial differential equation, which is associated to $m_{\mathbf{t}}^{*}$ in a simple way. As in $[3,4]$, in order to investigate the problem we employ the theory of distributions on the $n$-torus $\mathbf{T}^{n}$. We recall below some of our previous results and explain what is needed to generalize the argument used there so that we may deal with arbitrary distributions on $\mathbf{T}^{n}$. Let $\mathbf{D}$ denote the set of distributions on $\mathbf{T}^{n}$ (see [1], for example). A distribution $S \in \mathbf{D}$ is said to be of order $N$ if there exists a positive constant $c$ such that for all $u \in \mathbf{C}^{\infty}\left(\mathbf{T}^{n}\right)$ one has $|S(u)| \leq c \sup _{0 \leq|\mathbf{s}| \leq N}\left\|\partial^{\mathbf{s}} u\right\|_{\infty}$, where $\mathbf{s}=\left(s_{1}, \cdots, s_{n}\right) \in \mathbf{Z}_{\geq 0}^{n}$ is a multi-index, $|s|=\sum_{1 \leq i \leq n} s_{i}$, and $\partial^{\mathbf{s}} u=\partial^{|s|} u / \partial x_{1}^{s_{1}} \cdots \partial x_{n}^{s_{n}}$. We denote by $\mathbf{D}^{N}(N=$ $0,1,2, \cdots)$ the set of distributions of order at most $N$. Note that we have $\mathbf{D}=\bigcup_{N \geq 0} \mathbf{D}^{N}$. For any $S \in \mathbf{D}$, its Fourier coefficients are defined by $\hat{S}(\mathbf{k})=$ $S\left(e_{-\mathbf{k}}\right), \mathbf{k} \in \mathbf{Z}^{n}$, where $e_{\mathbf{m}} \in \mathbf{C}^{\infty}\left(\mathbf{T}^{n}\right), \mathbf{m} \in \mathbf{Z}^{n}$, is the function $\mathbf{x} \mapsto e^{i \mathbf{m} \cdot \mathbf{x}}$. It is known that if $S \in \mathbf{D}^{N}$, then its Fourier coefficient satisfies $\hat{S}(\mathbf{k})=O\left(|\mathbf{k}|^{N}\right)$ as $|\mathbf{k}| \rightarrow \infty$. Therefore the Fourier transform defines a linear isomorphism from $\mathbf{D}^{N}$ onto $\mathbf{A}^{N}$. Thus the set $\mathbf{A}^{0}$ (as well as its name) coincides with the set of bounded arrays investigated in [3, 4], which is the set of Fourier transforms of pseudomeasures. Furthermore note that the equality $\left(e_{-\mathbf{k}} S\right)^{\wedge}(\mathbf{p})=\hat{S}(\mathbf{p}+\mathbf{k})$ 
holds for any $\mathbf{p}=\left(p_{1}, \cdots, p_{n}\right) \in \mathbf{Z}^{n}$. Therefore for any window $\mathbf{t}$, we have

$$
\left(m_{\mathbf{t}}^{*} S\right)^{\wedge}(\mathbf{p})=\sum_{\mathbf{k} \in \mathbf{Z}^{n}} \mathbf{t}_{\mathbf{k}} \hat{S}(\mathbf{p}+\mathbf{k}), \quad \mathbf{p} \in \mathbf{Z}^{n} .
$$

Let $\mathbf{a}=\left(\mathbf{a}_{\mathbf{i}}\right) \in \mathbf{A}_{\mathbf{t}}^{\text {poly }}$ and let $A$ denote the Fourier transform of $\mathbf{a}$. Then it follows from (2.1) that

$$
\left(m_{\mathbf{t}}^{*} A\right)^{\wedge}(\mathbf{p})=\sum_{\mathbf{k} \in \mathbf{Z}^{n}} \mathbf{t}_{\mathbf{k}} \mathbf{a}_{\mathbf{p}+\mathbf{k}}
$$

which is equal to zero for any $\mathbf{p} \in \mathbf{Z}^{n}$, since $\mathbf{a}=\left(\mathbf{a}_{\mathbf{i}}\right) \in \mathbf{A}_{\mathbf{t}}^{\text {poly }}$. Hence, by the injectivity of Fourier transform, we have $m_{\mathbf{t}}^{*} A=0$. Thus we obtain the following:

Proposition 2.1. $\quad$ Notation being as above, we have $\operatorname{supp} A \subset V_{\mathbf{T}^{n}}\left(m_{\mathbf{t}}^{*}\right)$.

The following proposition plays a crucial role for our investigation in this paper.

Proposition $2.2([1,12.33])$. Let $S$ be a distribution whose support is a finite subset of $\mathbf{T}^{n}$, say $\left\{\mathbf{p}_{1}, \cdots, \mathbf{p}_{k}\right\}$. Then there exist finite subsets $\mathbf{I}_{j} \subset$ $\mathbf{Z}_{\geq 0}^{n}(j=1, \cdots, k)$ and a set of complex numbers $c_{j, \mathbf{s}}\left(j=1, \cdots, k ; \mathbf{s} \in \mathbf{I}_{j}\right\}$ such that

$$
S=\sum_{1 \leq j \leq k} \sum_{\mathbf{s} \in \mathbf{I}_{j}} c_{j, \mathbf{s}} \partial^{\mathbf{s}} \delta_{\mathbf{p}_{j}},
$$

where $\delta_{\mathbf{p}}$ is the Dirac delta function at the point $\mathbf{p} \in \mathbf{T}^{n}$.

Thus if we assume that $\#\left(V_{\mathbf{T}^{n}}\left(m_{\mathbf{t}}^{*}\right)\right)<\infty$, then the Fourier transform $A$ of an array $\mathbf{a} \in \mathbf{A}_{\mathbf{t}}^{\text {poly }}$ is expressed as a finite sum

$$
A=\sum_{\mathbf{p} \in V_{\mathbf{T}^{n}}\left(m_{\mathbf{t}}^{*}\right)} A(\mathbf{p}) \text { with } A(\mathbf{p})=\sum_{\mathbf{s} \in \mathbf{I}_{A(\mathbf{p})}} c_{\mathbf{p}, \mathbf{s}} \partial^{\mathbf{s}} \delta_{\mathbf{p}},
$$

where $\mathbf{I}_{A(\mathbf{p})}, \mathbf{p} \in V_{\mathbf{T}^{n}}\left(m_{\mathbf{t}}^{*}\right)$, is a finite subset of $\mathbf{Z}_{>0}^{n}$. Hence our task is to find the condition for $A$ of the above form such that $m_{\mathbf{t}}^{*} A=0$ holds. The following proposition shows that this condition can be dealt with separately for each $\mathbf{p} \in V_{\mathbf{T}^{n}}\left(m_{\mathbf{t}}^{*}\right)$.

Proposition 2.3. $\quad$ Notation being as above, suppose that $\#\left(V_{\mathbf{T}^{n}}\left(m_{\mathbf{t}}^{*}\right)\right)<$ $\infty$. Then the condition $m_{\mathbf{t}}^{*} A=0$ holds if and only if $m_{\mathbf{t}}^{*} A(\mathbf{p})=0$ holds for every $\mathbf{p} \in V_{\mathbf{T}^{n}}\left(m_{\mathbf{t}}^{*}\right)$. 
Proof. Let $N=\max \left\{|\mathbf{s}| ; \mathbf{s} \in \mathbf{I}_{A(\mathbf{p})}, \mathbf{p} \in V_{\mathbf{T}^{n}}\left(m_{\mathbf{t}}^{*}\right)\right\}$. For any $\mathbf{p} \in V_{\mathbf{T}^{n}}\left(m_{\mathbf{t}}^{*}\right)$, let $f_{\mathbf{p}} \in \mathbf{C}^{\infty}\left(\mathbf{T}^{n}\right)$ be a $\mathbf{C}^{\infty}$ function such that

$\left(\partial^{\mathbf{s}} f_{\mathbf{p}}\right)(\mathbf{q})=0$ for any $\mathbf{q} \in V_{\mathbf{T}^{n}}\left(m_{\mathbf{t}}^{*}\right)$ and $\mathbf{s}$ with $|\mathbf{s}| \leq N$, except that $f_{\mathbf{p}}(\mathbf{p})=1$.

(The existence of such a function is assured by the decomposition of unity.) Then we see that

$$
f_{\mathbf{p}}\left(\partial^{\mathbf{s}} \delta_{\mathbf{q}}\right)= \begin{cases}0, & \text { if } \mathbf{q} \in V_{\mathbf{T}^{n}}\left(m_{\mathbf{t}}^{*}\right)-\{\mathbf{p}\} \\ \partial^{\mathbf{s}} \delta_{\mathbf{p}}, & \text { if } \mathbf{q}=\mathbf{p}\end{cases}
$$

It follows that

$$
\begin{aligned}
f_{\mathbf{p}} A & =\sum_{\mathbf{q} \in V_{\mathbf{T}^{n}}\left(m_{\mathbf{t}}^{*}\right)} f_{\mathbf{p}} A(\mathbf{q})=\sum_{\mathbf{q} \in V_{\mathbf{T}^{n}}\left(m_{\mathbf{t}}^{*}\right)} f_{\mathbf{p}} \sum_{\mathbf{s} \in \mathbf{I}_{A(\mathbf{q})}} c_{\mathbf{q}, \mathbf{s}} \partial^{\mathbf{s}} \delta_{\mathbf{q}} \\
& =\sum_{\mathbf{s} \in \mathbf{I}_{A(\mathbf{p})}} c_{\mathbf{p}, \mathbf{s}} f_{\mathbf{p}}\left(\partial^{\mathbf{s}} \delta_{\mathbf{p}}\right)=\sum_{\mathbf{s} \in \mathbf{I}_{A(\mathbf{p})}} c_{\mathbf{p}, \mathbf{s}} \partial^{\mathbf{s}} \delta_{\mathbf{p}}=A(\mathbf{p}) .
\end{aligned}
$$

Hence if $m_{\mathbf{t}}^{*} A=0$, then $0=f_{\mathbf{p}} m_{\mathbf{t}}^{*} A=m_{\mathbf{t}}^{*} f_{\mathbf{p}} A=m_{\mathbf{t}}^{*} A(\mathbf{p})$. Since the converse is clear, this completes the proof.

This proposition reduces our task to the investigation of the space

$$
\left\{\sum_{\mathbf{s} \in I} c_{\mathbf{s}} \partial^{\mathbf{s}} \delta_{\mathbf{p}} ; I \text { is a finite subset of } \mathbf{Z}_{\geq 0}^{n}, c_{\mathbf{s}} \in \mathbf{C}, m_{\mathbf{t}}^{*}\left(\sum_{\mathbf{s} \in I} c_{\mathbf{s}} \partial^{\mathbf{s}} \delta_{\mathbf{p}}\right)=0\right\}
$$

for each $\mathbf{p} \in V_{\mathbf{T}^{n}}\left(m_{\mathbf{t}}^{*}\right)$. Through the Fourier transform, this translates to the space of zero-sum arrays as follows. Let $\mathbf{p}=\left(p_{1}, \cdots, p_{n}\right)=\left(e^{i a_{1}}, \cdots, e^{i a_{n}}\right) \in$ $\mathbf{T}^{n}$, and we take $w_{j}=e^{i x_{j}}-e^{i a_{j}}, 1 \leq j \leq n$, as a set of local coordinates at $\mathbf{p}$. Since $\partial_{w_{j}}=\left(-i / z_{j}\right) \partial_{x_{j}}$, where $z_{j}=e^{i x_{j}}(1 \leq j \leq n)$, we have

$$
\begin{aligned}
\left(\partial_{w_{j}} \delta_{\mathbf{p}}\right)(\mathbf{k}) & =\left(\left(-i / z_{j}\right) \partial_{x_{j}} \delta_{\mathbf{p}}\right)\left(e^{-i \mathbf{k} \cdot \mathbf{x}}\right) \\
& =\left(\partial_{x_{j}} \delta_{\mathbf{p}}\right)\left(-i e^{-i \mathbf{k} \cdot \mathbf{x}} / z_{j}\right) \\
& =\left(\partial_{x_{j}} \delta_{\mathbf{p}}\right)\left(-i e^{-i \mathbf{k} \cdot \mathbf{x}-i x_{j}}\right) \\
& =-(-i)\left(-i k_{j}-i\right) \delta_{\mathbf{p}}\left(e^{-i \mathbf{k} \cdot \mathbf{x}-i x_{j}}\right) \\
& =\left(k_{j}+1\right) e^{-i \mathbf{k} \cdot \mathbf{a}-i a_{j}} \\
& =\left(k_{j}+1\right) \mathbf{p}^{-\left(\mathbf{k}+\mathbf{e}_{j}\right)}
\end{aligned}
$$

where $\mathbf{e}_{j}(1 \leq j \leq n)$ denotes the $j$-th standard basis vector of $\mathbf{Z}^{n}$. In order to generalize this formula to the one for higher derivatives, we introduce the notation $a^{\bar{s}}=(a+1)(a+2) \cdots(a+s)$ for any integers $a$, $s$, and set $\mathbf{a}^{\overline{\mathbf{s}}}=$ 
$\prod_{1 \leq j \leq n} a_{j}^{\bar{s}_{j}}$. (The reader should be aware that this notation is different a little bit from that used in $[2,2.6])$. Then we have the following formula, which can be proved easily by induction.

Proposition 2.4. $\quad$ Notation being as above, we have $\left(\partial_{w_{1}}^{s_{1}} \cdots \partial_{w_{n}}^{s_{n}} \delta_{\mathbf{p}}\right)(\mathbf{k})=$ $\mathbf{k}^{\overline{\mathbf{s}}} \mathbf{p}^{-(\mathbf{k}+\mathbf{s})}$.

As a consequence, we obtain the following.

Theorem 2.5. $\quad$ Notation being as above, the equality $m_{\mathbf{t}}^{*}\left(\sum_{\mathbf{s} \in I} c_{\mathbf{s}} \partial^{\mathbf{s}} \delta_{\mathbf{p}}\right)=$ 0 holds if and only if $\left(\sum_{\mathbf{s} \in I} c_{\mathbf{s}} \mathbf{k}^{\overline{\mathbf{s}}} \mathbf{p}^{-(\mathbf{k}+\mathbf{s})}\right)_{\mathbf{k} \in \mathbf{Z}^{n}} \in \mathbf{A}_{\mathbf{t}}^{\text {poly }}$.

Furthermore we can get rid of dualizing operator "** in this formulation by the following simple trick. Given an array $\mathbf{a}=\left(\mathbf{a}_{\mathbf{i}}\right)_{\mathbf{i} \in \mathbf{Z}^{n}}$, we define a transposed array $\mathbf{a}^{*}$ by $\mathbf{a}^{*}=\left(\mathbf{a}_{-\mathbf{i}}\right)_{\mathbf{i} \in \mathbf{Z}^{n}}$, so that the equality $m_{\mathbf{t}^{*}}(\mathbf{z})=m_{\mathbf{t}}^{*}(\mathbf{z})$ holds. Note that for any window $\mathbf{t}$, we have

$$
\mathbf{A}_{\mathbf{t}}^{\text {poly }}=\left(\mathbf{A}_{\mathbf{t}^{*}}^{\text {poly }}\right)^{*},
$$

namely $\mathbf{a} \in \mathbf{A}_{\mathbf{t}}^{\text {poly }}$ if and only if $\mathbf{a}^{*} \in \mathbf{A}_{\mathbf{t}^{*}}^{\text {poly }}$, since $d_{\mathbf{t}^{*}-\mathbf{p}}\left(\mathbf{a}^{*}\right)=d_{\mathbf{t}+\mathbf{p}}(\mathbf{a})$ holds for any $\mathbf{p} \in \mathbf{Z}^{n}$. Therefore Theorem 2.5 is formulated solely in terms of the original window and its characteristic polynomial:

Theorem 2.6. $\quad$ Notation being as above, the equality $m_{\mathbf{t}}\left(\sum_{\mathbf{s} \in I} c_{\mathbf{s}} \partial^{\mathbf{s}} \delta_{\mathbf{p}}\right)=$ 0 holds if and only if $\left(\sum_{\mathbf{s} \in I} c_{\mathbf{s}}(-\mathbf{k})^{\overline{\mathbf{s}}} \mathbf{p}^{\mathbf{k}-\mathbf{s}}\right)_{\mathbf{k} \in \mathbf{Z}^{n}} \in \mathbf{A}_{\mathbf{t}}^{\text {poly }}$.

Thus our task turns out to be related to the annihilator of the Dirac delta function in the Weyl algebra. This is discussed in the next section.

\section{§3. Annihilator of the Delta Function}

As we have seen in the previous section, we can restrict our attention to the annihilator of $\delta_{\mathbf{p}}$ for each $\mathbf{p} \in V_{\mathbf{T}^{n}}\left(m_{\mathbf{t}}\right)$. This section is devoted to the local study of the annihilator and some related objects.

We take a system of local coordinates $\mathbf{w}=\left(w_{1}, \cdots, w_{n}\right)$ at $\mathbf{p}$, and let $\delta$ denote the Dirac delta function centered at the origin of $\mathbf{C}^{n}$. Let $W_{n}=$ $\mathbf{C}\left\langle w_{1}, \cdots, w_{n}, \partial_{1}, \cdots, \partial_{n}\right\rangle$ denote the Weyl algebra in $n$ variables, where $\partial_{i}=$ $\partial / \partial w_{i}, 1 \leq i \leq n$. We put $P_{n}=\mathbf{C}\left[w_{1}, \cdots, w_{n}\right], D_{n}=\mathbf{C}\left[\partial_{1}, \cdots, \partial_{n}\right]$, and call an element of the latter $\partial$-polynomial in order to discriminate it from the usual polynomial in the former. The Weyl algebra $W_{n}$ acts on $P_{n}$ through the usual differentiation. We denote this action by $F \cdot g$ for $F \in W_{n}, g \in P_{n}$, in 
order to distinguish it from the multiplication in the Weyl algebra, which is expressed simply by juxtaposition. One knows that the annihilator of $\delta$ in the Weyl algebra is equal to the left ideal $I_{n}=W_{n} w_{1}+\cdots+W_{n} w_{n}$. Therefore when we investigate the kernel of the multiplication operator $m_{g}: W_{n} \delta \rightarrow W_{n} \delta$, defined by $F \mapsto g F$ for $g \in P_{n}$, it is natural to consider its action through the quotient $W_{n} / I_{n}$. The following proposition and its corollary reveal an intimate connection between the kernel and the space of polynomial solutions of a certain PDE.

Proposition 3.1. Let $F_{+}, F_{-} \in H_{\text {or }} m_{\mathbf{C}-a l g}\left(W_{n}, W_{n}\right)$ denote the $\mathbf{C}$-algebra automorphisms of $W_{n}$ defined by

$$
\begin{gathered}
F_{+}\left(w_{i}\right)=\partial_{i}, \quad F_{+}\left(\partial_{i}\right)=w_{i}, \\
F_{-}\left(w_{i}\right)=-\partial_{i}, \quad F_{-}\left(\partial_{i}\right)=w_{i},
\end{gathered}
$$

for $1 \leq i \leq n$. Then for any $f \in \mathbf{C}\left[w_{1}, \cdots, w_{n}\right]$ and $D \in \mathbf{C}\left[\partial_{1}, \cdots, \partial_{n}\right]$, we have

$$
f D \equiv F_{+}\left(F_{-}(f) \cdot F_{-}(D)\right)\left(\bmod I_{n}\right) .
$$

Furthermore $f D \in I_{n}$ holds if and only if $F_{-}(f) \cdot F_{-}(D)=0$.

Proof. First we note that the relations

$$
w_{i} \partial_{i}^{s}=\partial_{i}^{s} w_{i}-s \partial_{i}^{s-1}, \quad s \geq 1, i=1, \cdots, n,
$$

hold in $W_{n}$. Therefore we have

$$
w_{i} \partial_{i}^{s} \equiv-s \partial_{i}^{s-1}\left(\bmod I_{n}\right), \quad s \geq 1, i=1, \cdots, n .
$$

Thus the multiplication operator $m_{w_{i}}$ acts, modulo $I_{n}$, on $D_{n}$ just like the negative of the "differentiation by $\partial_{i}$." The equality (3.1) is merely a translation of this fact into more precise form. As for the last assertion, note that $F_{-}(f)$. $F_{-}(D) \in \mathbf{C}\left[w_{1}, \cdots, w_{n}\right]$ and hence $F_{+}\left(F_{-}(f) \cdot F_{-}(D)\right) \in \mathbf{C}\left[\partial_{1}, \cdots, \partial_{n}\right]$. Since $I_{n} \cap \mathbf{C}\left[\partial_{1}, \cdots, \partial_{n}\right]=\{0\}$ by the linear independence of the derivatives of the Dirac delta function, it follows that if $f D \in I_{n}$, then $F_{-}(f) \cdot F_{-}(D)=0$. Since the converse is evident, this completes the proof.

This proposition has an important corollary. For any $f \in P_{n}, D \in D_{n}$, let

$$
\begin{gathered}
\operatorname{Diff}(f)=\left\{E \in D_{n} ; f E \cdot \delta=0\right\}, \\
\operatorname{Sol}(D)=\left\{g \in P_{n} ; D \cdot g=0\right\} .
\end{gathered}
$$

Then we have the following. 
Corollary 3.1.1. $\quad$ For any $f \in P_{n}, F_{-}$restricts to a linear isomorphism from Diff $(f)$ onto Sol $\left(F_{-}(f)\right)$.

Proof. Since the annihilator of $\delta$ is equal to $I_{n}$, the condition $E \in \operatorname{Diff}(f)$ holds if and only if $f E \in I_{n}$, which is equivalent to $F_{-}(f) \cdot F_{-}(E)=0$ by Proposition 3.1. This completes the proof.

Summarizing the results obtained in this and the previous sections, we obtain the following structure theorem on the space of zero-sum arrays. Let $G_{\mathbf{p}}: D_{n} \rightarrow \mathbf{A}^{\text {poly }}$ denote the linear map defined by $G_{\mathbf{p}}\left(\sum_{\mathbf{s}} c_{\mathbf{s}} \partial^{\mathbf{s}}\right)$ $=\left(\sum_{\mathbf{s}} c_{\mathbf{s}}(-\mathbf{k})^{\overline{\mathbf{s}}} \mathbf{p}^{\mathbf{k}-\mathbf{s}}\right)_{\mathbf{k} \in \mathbf{Z}^{n}}$ (see Theorem 2.6). Furthermore for any $D \in \mathbf{C}\left[\partial_{1}\right.$, $\left.\cdots, \partial_{n}\right]$, let $\operatorname{Sol}(D)_{\leq N}=\{f \in \operatorname{Sol}(D) ; \operatorname{deg} f \leq N\}$. Then we have the following.

Theorem 3.2. $\quad$ Suppose that $\#\left(V_{\mathbf{T}^{n}}\left(m_{\mathbf{t}}\right)\right)<\infty$. Then for each $\mathbf{p} \in$ $V_{\mathbf{T}^{n}}\left(m_{\mathbf{t}}\right)$, the composite map $G_{\mathbf{p}} \circ F_{-}^{-1}$ defines an isomorphism from $\operatorname{Sol}\left(F_{-}\left(m_{\mathbf{t}}^{\mathbf{p}}\right)\right)_{\leq N}$ onto its image in $\mathbf{A}_{\mathbf{t}}^{N}$, where $m_{\mathbf{t}}^{\mathbf{p}}(\mathbf{w})=m_{\mathbf{t}}(\mathbf{w}+\mathbf{p})$. Furthermore we have

$$
\mathbf{A}_{\mathbf{t}}^{N}=\underset{\mathbf{p} \in V_{\mathbf{T}^{n}}\left(m_{\mathbf{t}}\right)}{\oplus} G_{\mathbf{p}} \circ F_{-}^{-1}\left(\operatorname{Sol}\left(F_{-}\left(m_{\mathbf{t}}^{\mathbf{p}}\right)_{\leq N}\right)\right)
$$

and hence

$$
\mathbf{A}_{\mathbf{t}}^{\text {poly }}=\underset{\mathbf{p} \in V_{\mathbf{T}^{n}}\left(m_{\mathbf{t}}\right)}{\oplus} G_{\mathbf{p}} \circ F_{-}^{-1}\left(\operatorname{Sol}\left(F_{-}\left(m_{\mathbf{t}}^{\mathbf{p}}\right)\right)\right) .
$$

For actual computation of the space of zero-sum arrays, as will be done in the final section, it is convenient to reformulate the theorem in the form of an algorithm as follows:

Algorithm 3.3. Given a window $\mathbf{t}$, we can find every array in $\mathbf{A}_{\mathbf{t}}^{\text {poly }}$ through the following steps:

(A) Find $V_{\mathbf{T}^{n}}\left(m_{\mathbf{t}}\right)$.

(B) For $\mathbf{p} \in V_{\mathbf{T}^{n}}\left(m_{\mathbf{t}}\right)$, let $\mathbf{w}=\mathbf{z}-\mathbf{p}$ and put $m_{\mathbf{t}}^{\mathbf{p}}(\mathbf{w})=m_{\mathbf{t}}(\mathbf{w}+\mathbf{p})$.

(C) Put $D_{\mathbf{p}}=F_{-}\left(m_{\mathbf{t}}^{\mathbf{p}}\right)$ and determine $\operatorname{Sol}\left(D_{\mathbf{p}}\right)$.

(D) For any $f \in \operatorname{Sol}\left(D_{\mathbf{p}}\right)$, let $F_{-}^{-1}(f)=\sum c_{\mathbf{s}} \partial^{\mathrm{s}}$.

(E) Then $\sum c_{\mathbf{s}} \mathbf{k}^{\mathbf{s}} \mathbf{p}^{-(\mathbf{k}+\mathbf{s})} \in \mathbf{A}_{\mathbf{t}^{*}}^{\text {poly }}$, and hence $\sum c_{\mathbf{s}}(-\mathbf{k})^{\overline{\mathbf{s}}} \mathbf{p}^{\mathbf{k}-\mathbf{s}} \in \mathbf{A}_{\mathbf{t}}^{\text {poly }}$.

Thus it remains to investigate the step (C), namely the problem of finding polynomial solutions of linear partial differential equations with constant coefficients. This will be discussed in the following two sections. 


\section{§4. Dimension Formula}

In this section we derive a dimension formula for the space of polynomial solutions of linear partial differential equations with constant coefficients. As an application we show that the generating functions of the dimensions are always rational functions.

For any $D=\sum_{\mathbf{i} \in \mathbf{Z}_{\geq 0}^{n}} a_{\mathbf{i}} \partial^{\mathbf{i}} \in \mathbf{C}\left[\partial_{1}, \cdots, \partial_{n}\right]$, let $\operatorname{Supp}(D)=\left\{\mathbf{i} \in \mathbf{Z}_{\geq 0}^{n} ; a_{\mathbf{i}} \neq\right.$ $0\}$, and put $\operatorname{mdeg} D=\min \{|\mathbf{i}| ; \mathbf{i} \in \operatorname{Supp}(D)\}$. For any $f=\sum_{\mathbf{i} \in \mathbf{Z}_{\geq 0}^{n}} b_{\mathbf{i}} \mathbf{w}^{\mathbf{i}} \in$ $\mathbf{C}\left[w_{1}, \cdots, w_{n}\right]$, one can check that the constant term of $D \cdot f$ is equal to $\sum_{\mathbf{i} \in S u p p(D)} a_{\mathbf{i}} b_{\mathbf{i}} \mathbf{i}$ !, where $\mathbf{i} !=\prod_{1 \leq k \leq n} i_{k}$ !. Similarly one sees that the coefficient of $\mathbf{w}^{\mathbf{p}}\left(\mathbf{p} \in \mathbf{Z}_{\geq 0}^{n}\right)$ in $D \cdot f$ is equal to $\sum_{\mathbf{i} \in S u p p(D)} a_{\mathbf{i}} b_{\mathbf{i}+\mathbf{p}}(\mathbf{i}+\mathbf{p})$ !. Therefore if $f \in \operatorname{Sol}(D)$, then all of these coefficients must vanish. Let $E q(\mathbf{p})$ denote the equation $\sum_{\mathbf{i} \in \operatorname{Supp}(D)}(\mathbf{i}+\mathbf{p}) ! a_{\mathbf{i}} X_{\mathbf{i}+\mathbf{p}}=0$ arising in this way, and let $\operatorname{Suff}(\mathbf{p})=\operatorname{Supp}(D)+\mathbf{p}$, the set of suffices which appear in $E q(\mathbf{p})$. Let $\prec_{\text {lex }}$ denote the lexicographic order on $\mathbf{Z}_{\geq 0}^{n}$, and let $\prec$ denote the total order on $\mathbf{Z}_{\geq 0}^{n}$ defined by

$$
\mathbf{i} \prec \mathbf{j} \Leftrightarrow|\mathbf{i}|<|\mathbf{j}| \text { or }\left(|\mathbf{i}|=|\mathbf{j}| \text { and } \mathbf{i} \prec_{\text {lex }} \mathbf{j}\right) \text {. }
$$

Note that this order is compatible with the translation, namely, if $\mathbf{i} \prec \mathbf{j}$ and $\mathbf{k} \in \mathbf{Z}_{\geq 0}^{n}$, then $\mathbf{i}+\mathbf{k} \prec \mathbf{j}+\mathbf{k}$. Let $\operatorname{ind}(\mathbf{p}) \in \mathbf{Z}_{\geq 0}^{n}$ denote the minimum of the set $\operatorname{Suff}(\mathbf{p})$ with respect to the total order $\prec$. Then by the compatibility we have $\operatorname{ind}(\mathbf{p})=\min _{\prec}(\operatorname{Supp}(D))+\mathbf{p}$. Therefore the map ind $: \mathbf{Z}_{\geq 0}^{n} \rightarrow \mathbf{Z}_{\geq 0}^{n}$ is strictly increasing with respect to $\prec$. In particular, the equations $E q(\mathbf{p}), \mathbf{p} \in \mathbf{Z}_{\geq 0}^{n}$, are linearly independent. When $f=\sum_{\mathbf{i} \in \mathbf{Z}_{\geq 0}^{n}} b_{\mathbf{i}} \mathbf{x}^{\mathbf{i}} \in \operatorname{Sol}(D)_{\leq N}$, the set of nontrivial equations for the coefficients is detected as follows. Let $\Delta_{\leq N}^{n}=\left\{\mathbf{i} \in \mathbf{Z}_{\geq 0}^{n} ;|\mathbf{i}| \leq\right.$ $N\}$. The number of its elements is given by $\#\left(\Delta_{\leq N}^{n}\right)=\left(\begin{array}{c}n+N \\ n\end{array}\right)$. On the other hand, the equation $E q(\mathbf{p})=\sum_{\mathbf{i} \in S u p p(D)}(\mathbf{i}+\mathbf{p}) ! a_{\mathbf{i}} X_{\mathbf{i}+\mathbf{p}}=0$ for $f$ to belong to $\operatorname{Sol}(D)_{\leq N}$ is nontrivial if and only if $(\operatorname{Supp}(D)+\mathbf{p}) \cap \Delta_{\leq N}^{n} \neq \phi$. The latter condition holds precisely when $\mathbf{p} \in\left(\Delta_{\leq N}^{n}-S u p p(D)\right) \cap \mathbf{Z}_{\geq 0}^{n}$. (Here we set $A-B=\{\mathbf{i}-\mathbf{j} ;(\mathbf{i}, \mathbf{j}) \in A \times B\} \subset \mathbf{Z}^{n}$ for any pair $A, B$ of subsets of $\mathbf{Z}_{\geq 0}^{n}$.) Thus we obtain the following.

Proposition 4.1. Notation being as above, we have

(4.1) $\operatorname{dim} \operatorname{Sol}(D)_{\leq N}=\max \left\{\left(\begin{array}{c}n+N \\ n\end{array}\right)-\#\left(\left(\Delta_{\leq N}^{n}-\operatorname{Supp}(D)\right) \cap \mathbf{Z}_{\geq 0}^{n}\right), 0\right\}$.

Actually we do not need the whole $\operatorname{Supp}(D)$ to compute the right hand side, as is shown by the following.

Lemma 4.2. For any $\mathbf{i} \in \mathbf{Z}_{\geq 0}^{n}$, we have $\left(\Delta_{\leq N}^{n}-\mathbf{i}\right) \cap \mathbf{Z}_{\geq 0}^{n}=\Delta_{\leq N-|\mathbf{i}|}^{n}$. 
Proof. This follows from the following computation:

$$
\begin{aligned}
\left(\Delta_{\leq N}^{n}-\mathbf{i}\right) \cap \mathbf{Z}_{\geq 0}^{n} & =\left\{\mathbf{p} \in \mathbf{Z}_{\geq 0}^{n} ; \mathbf{p}+\mathbf{i} \in \Delta_{\leq N}^{n}\right\} \\
& =\left\{\mathbf{p} \in \mathbf{Z}_{\geq 0}^{n} ;|\mathbf{p}+\mathbf{i}| \leq N\right\} \\
& =\left\{\mathbf{p} \in \mathbf{Z}_{\geq 0}^{n} ; \sum_{k \in[1, n]}\left(p_{k}+i_{k}\right) \leq N\right\} \\
& =\left\{\mathbf{p} \in \mathbf{Z}_{\geq 0}^{n} ; \sum_{k \in[1, n]} p_{k} \leq N-\sum_{k \in[1, n]} i_{k}\right\} \\
& =\left\{\mathbf{p} \in \mathbf{Z}_{\geq 0}^{n} ;|p| \leq N-|i|\right\} \\
& =\Delta_{\leq N-|\mathbf{i}| \cdot}^{n} .
\end{aligned}
$$

This lemma together with Proposition 4.1 implies the following formula.

Theorem 4.3. $\quad \operatorname{dim} \operatorname{Sol}(D)_{\leq N}=\left(\begin{array}{c}n+N \\ n\end{array}\right)-\left(\begin{array}{c}n+N-\operatorname{mdeg} D \\ n\end{array}\right)$.

Proof. Since $\Delta_{\leq M}^{n} \subset \Delta_{\leq N}^{n}$ whenever $M \leq N$, it follows from Lemma 4.2 that $\left(\Delta_{\leq N}^{n}-\operatorname{Supp}(\bar{D})\right) \cap \mathbf{Z}_{\geq 0}^{n}=\Delta_{\leq N-\operatorname{mdeg} D}^{n}$. Inserting this equality into the right hand side of (4.1), we finish the proof.

Corollary 4.3.1. If $D \in D_{n}$ contains a nonzero constant term, then $\operatorname{Sol}(D)_{\leq N}=\{0\}$

Theorem 4.3 gives through Theorem 3.2 a dimension formula for the space of zero-sum arrays. Let $V_{\mathbf{T}^{n}}\left(m_{\mathbf{t}}\right)=\left\{\mathbf{p}_{1}, \cdots, \mathbf{p}_{\ell}\right\}$, and let $\mathbf{w}=\mathbf{z}-\mathbf{p}_{k}, 1 \leq k \leq$ $\ell$, the local coordinate at $\mathbf{p}_{k}$. Let $m^{\mathbf{p}_{k}} \in \mathbf{C}\left[w_{1}, \cdots, w_{n}\right]$ denote the translated characteristic polynomial defined by $m_{\mathbf{t}}^{\mathbf{p}_{k}}(\mathbf{w})=m_{\mathbf{t}}\left(\mathbf{w}+\mathbf{p}_{k}\right)$. We define $m$ degree mdeg $f$ of a polynomial $f=\sum_{\mathbf{i} \in \mathbf{Z}_{>0}^{n}} a_{\mathbf{i}} \mathbf{w}^{i} \in \mathbf{C}\left[w_{1}, \cdots, w_{n}\right]$ to be equal to $\min \left\{|\mathbf{i}| ; a_{\mathbf{i}} \neq 0\right\}$, so that the equality mdeg $f=\operatorname{mdeg} F_{-}(f)$ holds. Then by Theorem 3.2 and Theorem 4.3, we have the following dimension formula.

Theorem 4.4. Notation being as above, we have

$$
\operatorname{dim}_{\mathbf{C}} \mathbf{A}_{\mathbf{t}}^{N}=\sum_{1 \leq k \leq \ell}\left(\left(\begin{array}{c}
n+N \\
n
\end{array}\right)-\left(\begin{array}{c}
n+N-\operatorname{mdeg} m_{\mathbf{t}}^{\mathbf{p}_{k}} \\
n
\end{array}\right)\right) .
$$

Next we focus on the set of solutions in homogeneous polynomials. Let $H \operatorname{Sol}(D)_{N}=\{f \in \operatorname{Sol}(D) ; \mathrm{f}$ is homogeneous with $\operatorname{deg} f=N\}$. First we consider the case when $D$ is homogeneous as $\partial$-polynomial. 
Theorem 4.5. Let $D$ be a homogeneous $\partial$-polynomial of degree $d$.

Then we have

$$
\operatorname{dim} H \operatorname{Sol}(D)_{N}=\left(\begin{array}{c}
n-1+N \\
n-1
\end{array}\right)-\left(\begin{array}{c}
n-1+N-d \\
n-1
\end{array}\right) .
$$

Proof. We use this time $\Delta_{=N}^{n}=\left\{\mathbf{i} \in \mathbf{Z}_{\geq 0}^{n} ;|\mathbf{i}|=N\right\}$ instead of $\Delta_{\leq N}^{n}$. The number of its elements is equal to $\#\left(\Delta_{=N}^{n}\right)=\#\left(\Delta_{\leq N}^{n-1}\right)=\left(\begin{array}{c}n-1+N \\ n-1\end{array}\right)$. Furthermore we see that the equation $E q(\mathbf{p})=\sum_{\mathbf{i} \in S u p p(D)}(\mathbf{i}+\mathbf{p}) ! \mathbf{a}_{\mathbf{i}} X_{\mathbf{i}+\mathbf{p}}=0$ for $f$ to belong to $\operatorname{HSol}(D)_{N}$ is nontrivial if and only if $\mathbf{p} \in \Delta_{=N-d}^{n}$. Therefore we have

$$
\operatorname{dim} H \operatorname{Sol}(D)_{N}=\left(\begin{array}{c}
n-1+N \\
n-1
\end{array}\right)-\left(\begin{array}{c}
n-1+N-d \\
n-1
\end{array}\right) .
$$

(Note that $\left(\begin{array}{c}n-1+N-d \\ n-1\end{array}\right)$ is defined to be zero when $N<d$.) This completes the proof.

Let us introduce the generating functions for the dimensions of polynomial (resp. homogeneous polynomial) solutions by

$$
\begin{aligned}
S_{D}(t) & =\sum_{N \geq 0}\left(\operatorname{dim} \operatorname{Sol}(D)_{\leq N}\right) t^{N} \\
H S_{D}(t) & =\sum_{N \geq 0}\left(\operatorname{dim} H \operatorname{Sol}(D)_{N}\right) t^{N} .
\end{aligned}
$$

Then the above results show that they have impressive forms:

Proposition 4.6. Notation being as above, we have

$$
S_{D}(t)=\frac{1-t^{\operatorname{mdeg} D}}{(1-t)^{n+1}} .
$$

When $D$ is homogeneous as a $\partial$-polynomial, then we have

$$
H S_{D}(t)=\frac{1-t^{\operatorname{deg} D}}{(1-t)^{n}} .
$$

Proof. By the (generalized) binomial expansion, we have $\sum_{N \geq 0}\left(\begin{array}{c}n+N \\ n\end{array}\right) t^{N}=$ $\frac{1}{(1-t)^{n+1}}$. It follows that

$$
\begin{aligned}
\sum_{N \geq 0}\left(\begin{array}{c}
n+N-\operatorname{mdeg} D \\
n
\end{array}\right) t^{N} & =\sum_{N \geq \operatorname{mdeg} D}\left(\begin{array}{c}
n+N-\operatorname{mdeg} D \\
n
\end{array}\right) t^{N} \\
& =t^{\operatorname{mdeg} D} \sum_{N \geq \operatorname{mdeg} D}\left(\begin{array}{c}
n+N-\operatorname{mdeg} D \\
n
\end{array}\right) t^{N-\operatorname{mdeg} D} \\
& =\frac{t^{\operatorname{mdeg} D}}{(1-t)^{n+1}}
\end{aligned}
$$


Hence Theorem 4.3 implies the formula (4.2). Similarly Theorem 4.5 shows the validity of (4.3). This completes the proof.

We examine our results through several examples.

Example 4.7.1. $\quad$ Let $D_{\text {harmonic }}=\nabla_{2}^{2}=\partial_{1}^{2}+\partial_{2}^{2}$. It is homogeneous with mdeg $D_{\text {harmonic }}=2$. Therefore Theorems 4.3 and 4.5 imply that

$$
\begin{aligned}
\operatorname{dim} \operatorname{Sol}\left(D_{\text {harmonic }}\right)_{N} & =\left(\begin{array}{c}
2+N \\
2
\end{array}\right)-\left(\begin{array}{c}
2+N-2 \\
2
\end{array}\right) \\
& =\left(\begin{array}{c}
N+2 \\
2
\end{array}\right)-\left(\begin{array}{c}
N \\
2
\end{array}\right)=2 N+1, \\
\operatorname{dim} H \operatorname{Sol}\left(D_{\text {harmonic }}\right)_{N} & =\left(\begin{array}{c}
1+N \\
1
\end{array}\right)-\left(\begin{array}{c}
1+N-2 \\
1
\end{array}\right) \\
& =\left(\begin{array}{c}
N+1 \\
1
\end{array}\right)-\left(\begin{array}{c}
N-1 \\
1
\end{array}\right)= \begin{cases}2, & \text { if } N \geq 1, \\
1, & \text { if } N=0 .\end{cases}
\end{aligned}
$$

(Recall the convention that $\left(\begin{array}{l}n \\ k\end{array}\right)=0$ when $k \notin[0, n]$.) Furthermore by Proposition 4.6, the generating functions are found to be

$$
\begin{gathered}
S_{D_{\text {harmonic }}}(t)=\frac{1-t^{2}}{(1-t)^{3}}=\frac{1+t}{(1-t)^{2}}, \\
H S_{D_{\text {harmonic }}}(t)=\frac{1-t^{2}}{(1-t)^{2}}=\frac{1+t}{1-t} .
\end{gathered}
$$

Example 4.7.2. Let $D_{\text {biharmonic }}=\left(\nabla_{2}^{2}\right)^{2}=\partial_{1}^{4}+2 \partial_{1}^{2} \partial_{2}^{2}+\partial_{2}^{4}$. Since $D_{\text {biharmonic }}$ is homogeneous and mdeg $D_{\text {biharmonic }}=4$, it follows Theorems 4.3 and 4.5 that

$$
\begin{aligned}
& \operatorname{dim} \operatorname{Sol}\left(D_{\text {biharmonic }}\right)_{\leq N}=\left(\begin{array}{c}
2+N \\
2
\end{array}\right)-\left(\begin{array}{c}
2+N-4 \\
2
\end{array}\right)=\left(\begin{array}{c}
N+2 \\
2
\end{array}\right)-\left(\begin{array}{c}
N-2 \\
2
\end{array}\right) \\
&= \begin{cases}4 N-2, & \text { if } N \geq 2, \\
3, & \text { if } N=1, \\
1, & \text { if } N=0 .\end{cases} \\
& \operatorname{dim} H \operatorname{Sol}\left(D_{\text {biharmonic }}\right)_{N}=\left(\begin{array}{c}
1+N \\
1
\end{array}\right)-\left(\begin{array}{c}
1+N-4 \\
1
\end{array}\right)=\left(\begin{array}{c}
N+1 \\
1
\end{array}\right)-\left(\begin{array}{c}
N-3 \\
1
\end{array}\right) \\
&= \begin{cases}4 & \text { if } N \geq 3, \\
3, & \text { if } N=2, \\
2, & \text { if } N=1, \\
1, & \text { if } N=0 .\end{cases}
\end{aligned}
$$


Furthermore the formulas (4.2) and (4.3) become

$$
\begin{gathered}
S_{D_{\text {biharmonic }}(t)}=\frac{1-t^{4}}{(1-t)^{3}}=\frac{1+t+t^{2}+t^{3}}{(1-t)^{2}}, \\
H S_{D_{\text {biharmonic }}}(t)=\frac{1-t^{4}}{(1-t)^{2}}=\frac{1+t+t^{2}+t^{3}}{1-t} .
\end{gathered}
$$

Example 4.7.3. Let $D_{\text {corner }}=\partial_{1}+\cdots+\partial_{n}$. (The name comes from the shape of the corresponding window. See Subsection 6.1.) Since $D_{\text {corner }}$ is homogeneous with mdeg $D_{\text {corner }}=1$, Theorem 4.5 and Proposition 4.6 imply that

$$
\begin{aligned}
\operatorname{dim} H \operatorname{Sol}\left(D_{\text {corner }}\right)_{N}= & \max \left\{\left(\begin{array}{c}
n-1+N \\
n-1
\end{array}\right)-\left(\begin{array}{c}
n-1+N-1 \\
n-1
\end{array}\right), 0\right\} \\
= & \begin{cases}\left(\begin{array}{c}
n-2+N \\
n-2
\end{array}\right), & n \geq 2 \\
1, & n=1, N=0 \\
0, & n=1, N \geq 1\end{cases} \\
& H S_{D}(t)=\frac{1}{(1-t)^{n-1}}
\end{aligned}
$$

Next we investigate the set of homogeneous solutions of non-homogeneous PDE's.

Theorem 4.8. Let $D$ be a non-homogeneous $\partial$-polynomial and let $D=$ $\sum_{i \in[1, k]} D_{d_{i}}$ be its decomposition into homogeneous parts with $\operatorname{deg} D_{d_{i}}=d_{i}, i \in$ $[1, k]$. Then $H \operatorname{Sol}(D)_{N}=\{0\}$ for sufficiently large $N$ if and only if $V\left(F_{+}\left(D_{d_{1}}\right)\right.$, $\left.\cdots, F_{+}\left(D_{d_{k}}\right)\right)=\{\mathbf{0}\} \in \mathbf{A}^{n}$, where $V\left(F_{+}\left(D_{d_{1}}\right), \cdots, F_{+}\left(D_{d_{k}}\right)\right)$ denotes the zero locus of the polynomials $F_{+}\left(D_{d_{1}}\right), \cdots, F_{+}\left(D_{d_{k}}\right) \in k\left[w_{1}, \cdots, w_{n}\right]$ (see Proposition 3.1 for the definition of $\left.F_{+}\right)$.

Proof. First we note that $h \in H \operatorname{Sol}(D)_{N}$ if and only if $D_{d_{i}} \cdot h=0$ for any $i \in[1, k]$. We begin with the proof of the if-part of the theorem.

If-part: Let $f_{i}=F_{+}\left(D_{d_{i}}\right), i \in[1, k]$. Then we have $V\left(f_{1}, \cdots, f_{k}\right)=$ $\{\mathbf{0}\}$, and hence $I\left(V\left(f_{1}, \cdots, f_{k}\right)\right)=I(\{\mathbf{0}\})=\left(w_{1}, \cdots, w_{n}\right)$. It follows from Hilbert's Nullstellensatz that $\sqrt{\left(f_{1}, \cdots, f_{k}\right)}=\left(w_{1}, \cdots, w_{n}\right)$. Therefore there exists an integer $M$ such that $w_{j}^{M} \in\left(f_{1}, \cdots, f_{k}\right)$ for any $j \in[1, n]$. It follows that when $N>k(M-1)$, every monomial $\mathbf{w}^{\mathbf{i}}$ of degree $N$ belongs to the ideal $\left(f_{1}, \cdots, f_{k}\right)$ by pigeonhole principle. Therefore for any $\mathbf{i} \in \Delta_{\geq N}^{n}=\{\mathbf{i} \in$ $\left.\mathbf{Z}_{\geq 0}^{n} ;|\mathbf{i}| \geq N\right\}$ with $N>k(M-1)$, there exists a set of polynomial $g_{i}, i \in$ $[1, k]$ such that $\partial^{\mathbf{i}}=F_{+}\left(\mathbf{w}^{\mathbf{i}}\right)$ is expressed as $\partial^{\mathbf{i}}=\sum_{i \in[1, k]} F_{+}\left(g_{i}\right) F_{+}\left(f_{i}\right)=$ 
$\sum_{i \in[1, k]} F_{+}\left(g_{i}\right) F_{+}\left(F_{+} D_{d_{i}}\right)=\sum_{i \in[1, k]} F_{+}\left(g_{i}\right) D_{d_{i}}$. Hence we see that if $h \in$ $H \operatorname{Sol}(D)_{N}$ with $N>k(M-1)$, then $\partial^{\mathrm{i}} \cdot h=\sum_{i \in[1, k]} F_{+}\left(g_{i}\right) D_{d_{i}} \cdot h=0$, which implies that $h=0$. Thus we finish the proof of the if-part.

Only-if-part: Suppose that $V=V\left(F_{+}\left(D_{d_{1}}\right), \cdots, F_{+}\left(D_{d_{k}}\right)\right) \supsetneqq\{\mathbf{0}\}$ and let $\mathbf{0} \neq\left(a_{1}, \cdots, a_{n}\right) \in V$. Since all of $F_{+}\left(D_{d_{i}}\right), i \in[1, k]$, are homogeneous, it follows that the zero locus $V$ contains the line $\ell=\left\{\left(t a_{1}, \cdots, t a_{n}\right) ; t \in \mathbf{C}\right\}$. Therefore we have $I(V) \subset I(\ell)=\left(a_{2} w_{1}-a_{1} w_{2}, a_{3} w_{1}-a_{1} w_{3}, \cdots, a_{n} w_{1}-a_{1} w_{n}\right)$. Since $f_{i} \in \sqrt{\left(f_{1}, \cdots, f_{k}\right)}=I(V)$, it follows that for any $i \in[1, k]$, there exists a set of polynomials $s_{j}, j \in[1, n]$, such that $f_{i}=\sum_{j \in[1, n]} s_{j}\left(a_{j} w_{1}-a_{1} w_{j}\right)$. On the other hand, if we put $h=\sum_{j \in[1, n]} a_{j} w_{j}$, then for any positive integer $p$, we have $\left(a_{j} \partial_{1}-a_{1} \partial_{j}\right) \cdot h^{p}=p h^{p-1}\left(\left(a_{j} \partial_{1}-a_{1} \partial_{j}\right) \cdot h\right)=p h^{p-1}\left(a_{j} a_{1}-a_{1} a_{j}\right)=0$. Therefore we see that

$$
\begin{aligned}
D_{d_{i}} \cdot h^{p} & =F_{+}\left(f_{i}\right) \cdot h=\left(\sum_{j \in[1, n]} F_{+}\left(s_{j}\right) F_{+}\left(a_{j} w_{1}-a_{1} w_{j}\right)\right) \cdot h \\
& =\sum_{j \in[1, n]} F_{+}\left(s_{j}\right)\left(a_{j} \partial_{1}-a_{1} \partial_{j}\right) \cdot h=0
\end{aligned}
$$

holds for any positive integer $p$. It follows that $h^{p} \in H \operatorname{Sol}(D)_{p}, p \geq 1$. This completes the proof of the only-if-part, and at the same time that of Theorem 4.8 .

The next example deals with a non-homogeneous $\partial$-polynomial.

Example 4.8.1. Let $D_{\text {harmonic }}=\partial_{1}^{2}+\partial_{2}^{2}-\partial_{1}^{2} \partial_{2}-\partial_{1} \partial_{2}^{2}$. As we will see later in Subsection 6.3, this corresponds to a window related to a class of discrete harmonic functions. Since mdeg $D_{\text {harmonic }}=2$, the generating function $S_{D_{\text {harmonic }}}(t)$ is the same as in Example 4.7.1. In order to investigate homogeneous solutions, we employ the notation in the proof of Theorem 4.8. Let $D=D_{\text {harmonic }}=D_{2}+D_{3}$, where

$$
D_{2}=\partial_{1}^{2}+\partial_{2}^{2}, D_{3}=-\partial_{1}^{2} \partial_{2}-\partial_{1} \partial_{2}^{2}
$$

and let

$$
f_{1}=F_{+}\left(D_{2}\right)=w_{1}^{2}+w_{2}^{2}, f_{2}=F_{+}\left(D_{3}\right)=-w_{1}^{2} w_{2}-w_{1} w_{2}^{2} .
$$

Since one can check that $V\left(f_{1}, f_{2}\right)=\{\mathbf{0}\}$, it follows from Theorem 4.8 that 
$H \operatorname{Sol}(D)_{N}=\{0\}$ for sufficiently large $N$. Actually we see that

$$
\begin{aligned}
w_{1}^{4} & =\left(\left(2 w_{1}^{2}+w_{1} w_{2}\right) f_{1}+\left(w_{1}+w_{2}\right) f_{2}\right) / 2, \\
w_{1}^{3} w_{2} & =\left(w_{1} w_{2} f_{1}+\left(-w_{1}+w_{2}\right) f_{2}\right) / 2, \\
w_{1}^{2} w_{2}^{2} & =\left(-w_{1} w_{2} f_{1}+\left(-w_{1}-w_{2}\right) f_{2}\right) / 2, \\
w_{1} w_{2}^{3} & =\left(w_{1} w_{2} f_{1}+\left(w_{1}-w_{2}\right) f_{2}\right) / 2, \\
w_{2}^{4} & =\left(\left(w_{1} w_{2}+2 w_{2}^{2}\right) f_{1}+\left(w_{1}+w_{2}\right) f_{2}\right) / 2 .
\end{aligned}
$$

It follows that we have an inclusion of ideals $\left(w_{1}, w_{2}\right)^{4} \subset\left(f_{1}, f_{2}\right)$, hence by the argument used in the proof of Theorem 4.8, we see that $H \operatorname{Sol}(D)_{N}=\{0\}$ for $N \geq 4$. Since one can check easily that $\operatorname{dim} H \operatorname{Sol}(D)_{0}=1, \operatorname{dim} H \operatorname{Sol}(D)_{1}=$ 2, dim $H \operatorname{Sol}(D)_{2}=2, \operatorname{dim} H \operatorname{Sol}(D)_{3}=1$ (see Example 5.5.3), we see that the generating function $H S_{D_{\text {harmonic }}}(t)=1+2 t+2 t^{2}+t^{3}$, a polynomial of degree three.

Remark 4.8.2. When $D=\partial_{1} \partial_{2}+\partial_{2}$, we see that the corresponding zero locus is $V\left(F_{+}\left(D_{2}\right), F_{+}\left(D_{1}\right)\right)=V\left(w_{1} w_{2}, w_{2}\right)=V\left(w_{2}\right) \neq\{\mathbf{0}\}$. Hence it follows from Theorem 4.8 that there are infinitely many independent homogeneous solutions. Actually one can show that $H \operatorname{Sol}(D)_{N}=\left\langle w_{1}^{N}\right\rangle_{\mathbf{C}}$ for any $N$. Indeed, this phenomenon is also related to the fact that $D$ is factored as $D=\left(\partial_{1}+1\right) \partial_{2}$ and $\operatorname{Sol}\left(\partial_{2}\right)=\mathbf{C}\left[w_{1}\right]$. We must, however, be careful not to conclude that the reducibility always implies the infiniteness of homogeneous solutions. In fact, for $D=\left(\partial_{1} \partial_{2}+\partial_{1}+\partial_{2}\right)^{2}$, we have $V\left(F_{+}\left(D_{4}\right), F_{+}\left(D_{3}\right), F_{+}\left(D_{2}\right)\right)=$ $V\left(w_{1}^{2} w_{2}^{2}, 2 w_{1} w_{2}\left(w_{1}+w_{2}\right),\left(w_{1}+w_{2}\right)^{2}\right)=\{\mathbf{0}\}$, and hence Theorem $4.8 \mathrm{im}-$ plies that $\operatorname{HSol}(D)_{N}=\{0\}$ for sufficiently large $N$. On the other hand, for $D=\partial_{1} \partial_{2}^{2}+\partial_{1} \partial_{3}+\partial_{3}$, which is easily seen to be irreducible, we see that $V\left(F_{+}\left(D_{3}\right), F_{+}\left(D_{2}\right), F_{+}\left(D_{1}\right)\right)=V\left(w_{1} w_{2}^{2}, w_{1} w_{3}, w_{3}\right)$ contains a point $(1,0,0) \in \mathbf{A}^{3}$, and hence Theorem 4.8 implies that there are infinitely many independent homogeneous solutions for $D$.

\section{§5. Polynomial Solutions of PDE}

In this section we establish a method which derives a polynomial solution of a PDE from that of a certain PDE of lesser variables.

One of standard methods for finding polynomial solutions of PDE is to translate the problem into solving a system of linear difference equations on the unknown coefficients of the solutions. Here we propose an inductive procedure, which enables one to find polynomial solutions of PDE from those for lesser variables. Observe that for any $D \in \mathbf{C}\left[\partial_{1}, \cdots, \partial_{n}\right]$, we have $\partial_{i}(\operatorname{Sol}(D)) \subset$ 
$\operatorname{Sol}(D)(1 \leq i \leq n)$. Therefore it is natural to consider what occurs if we integrate an arbitrary solution. Let $S_{k} \in \operatorname{End}_{\mathbf{C}}\left(P_{n}\right)(1 \leq k \leq n)$ denote the linear map defined by

$$
S_{k}\left(\sum_{\mathbf{i} \in \mathbf{Z}_{\geq 0}^{n}} a_{\mathbf{i}} \mathbf{w}^{\mathbf{i}}\right)=\sum_{\mathbf{i} \in \mathbf{Z}_{\geq 0}^{n}} \frac{1}{i_{k}+1} a_{\mathbf{i}} \mathbf{w}^{\mathbf{i}+\mathbf{e}_{k}},
$$

where $\mathbf{e}_{k}(1 \leq k \leq n)$ denotes the $k$-th standard basis of $\mathbf{Z}^{n}$.

Proposition 5.1. The following commutator relations hold in $\operatorname{End}_{\mathbf{C}}\left(P_{n}\right)$ :

$$
\begin{gathered}
\partial_{k} S_{\ell}-S_{\ell} \partial_{k}=0, \text { if } k \text { and } \ell \text { are distinct, } \\
\partial_{k} S_{k}-S_{k} \partial_{k}=p_{\hat{k}}, \quad 1 \leq k \leq n,
\end{gathered}
$$

where $p_{\hat{k}}$ is defined by $\left(p_{\hat{k}} f\right)\left(w_{1}, \cdots, w_{n}\right)=f\left(w_{1}, \cdots, w_{k-1}, 0, w_{k+1}, \cdots, w_{n}\right)$.

Proof. The first equality can be checked easily. As for the second one, let $f=\sum_{\mathbf{i} \in \mathbf{Z}_{\geq 0}^{n}} a_{\mathbf{i}} \mathbf{w}^{\mathbf{i}}$. Then we have

$$
\begin{aligned}
S_{k} \partial_{k}(f) & =S_{k}\left(\sum_{\mathbf{i} \in \mathbf{Z}_{\geq 0}^{n}+\mathbf{e}_{k}} i_{k} a_{\mathbf{i}} \mathbf{w}^{\mathbf{i}-\mathbf{e}_{k}}\right)=\sum_{\mathbf{i} \in \mathbf{Z}_{\geq 0}^{n}+\mathbf{e}_{k}} a_{\mathbf{i}} \mathbf{w}^{\mathbf{i}} \\
& =\sum_{\mathbf{i} \in \mathbf{Z}_{\geq 0}^{n}} a_{\mathbf{i}} \mathbf{w}^{\mathbf{i}}-\sum_{\substack{\mathbf{i} \in \mathbf{Z}_{\geq 0}^{n} \\
i_{k}=0}} a_{\mathbf{i}} \mathbf{w}^{\mathbf{i}}=f-p_{\hat{k}}(f) .
\end{aligned}
$$

Since $\partial_{k} S_{k}=i d$, this implies (5.2). This completes the proof.

Corollary 5.1.1. Let $m$ be an arbitrary nonnegative integer. Then for any pair of distinct integers $k, \ell \in[1, n]$, we have

$$
\partial_{k}^{m} S_{\ell}=S_{\ell} \partial_{k}^{m}
$$

On the other hand, for any $k \in[1, n]$, we have

$$
\partial_{k}^{m} S_{k}= \begin{cases}S_{k} \partial_{k}^{m}+p_{\hat{k}} \partial_{k}^{m-1}, & \text { if } m \geq 1 \\ S_{k} \partial_{k}^{m}, & \text { if } m=0\end{cases}
$$

Proof. The first assertion is clear. As for the second assertion, when $m=2$, noting that $\partial_{k} p_{\hat{k}}=0$, we see from (5.2) that

$$
\partial_{k}^{2} S_{k}=\partial_{k}\left(S_{k} \partial_{k}+p_{\hat{k}}\right)=\partial_{k} S_{k} \partial_{k}=\left(S_{k} \partial_{k}+p_{\hat{k}}\right) \partial_{k}=S_{k} \partial_{k}^{2}+p_{\hat{k}} \partial_{k},
$$


For general $m$, one can prove (5.4) by induction. This finishes the proof.

From this corollary, we obtain the following.

Proposition 5.2. For any $D=\sum_{\mathbf{i} \in \mathbf{Z}_{\geq 0}^{n}} b_{\mathbf{i}} \partial^{\mathbf{i}} \in \mathbf{C}\left[\partial_{1}, \cdots, \partial_{n}\right]$ and for any integer $k$ with $1 \leq k \leq n$, let $D_{k}^{-}=\sum_{\mathbf{i} \in \mathbf{Z}_{>0}^{n}+\mathbf{e}_{k}} b_{\mathbf{i}} \partial^{\mathbf{i}-\mathbf{e}_{k}}$. Then we have

$$
D S_{k}=S_{k} D+p_{\hat{k}} D_{k}^{-}
$$

Proof. It follows from Corollary 5.1.1 that

$$
\begin{aligned}
D S_{k} & =\sum_{\mathbf{i} \in \mathbf{Z}_{\geq 0}^{n}} b_{\mathbf{i}} \partial^{\mathbf{i}} S_{k} \\
& =\sum_{\mathbf{i} \in \mathbf{Z}_{\geq 0}^{n}} b_{\mathbf{i}} \partial_{k}^{i_{k}} S_{k} \partial^{\mathbf{i}-i_{k} \mathbf{e}_{k}} \\
& =\sum_{\substack{\mathbf{i} \in \mathbf{Z}_{\geq 0}^{n} \\
i_{k} \geq 1}} b_{\mathbf{i}} \partial_{k}^{i_{k}} S_{k} \partial^{\mathbf{i}-i_{k} \mathbf{e}_{k}}+\sum_{\substack{\mathbf{i} \in \mathbf{Z}_{\geq 0}^{n} \\
i_{k}=0}} b_{\mathbf{i}} \partial_{k}^{i_{k}} S_{k} \partial^{\mathbf{i}-i_{k} \mathbf{e}_{k}} \\
& =\sum_{\substack{\mathbf{i} \in \mathbf{Z}_{\geq 0}^{n} \\
i_{k} \geq 1}} b_{\mathbf{i}}\left(S_{k} \partial_{k}^{i_{k}}+p_{\hat{k}} \partial_{k}^{i_{k}-1}\right) \partial^{\mathbf{i}-i_{k} \mathbf{e}_{k}}+\sum_{\substack{\mathbf{i} \in \mathbf{Z}_{\geq 0}^{n} \\
i_{k}=0}} b_{\mathbf{i}} S_{k} \partial^{\mathbf{i}} \\
& =\sum_{\substack{\mathbf{i} \in \mathbf{Z}_{\geq 0}^{n} \\
i_{k} \geq 1}} b_{\mathbf{i}}\left(S_{k} \partial^{\mathbf{i}}+p_{\hat{k}} \partial^{\mathbf{i}-\mathbf{e}_{k}}\right)+\sum_{\substack{\mathbf{i} \in \mathbf{Z}_{\geq 0}^{n} \\
i_{k}=0}} b_{\mathbf{i}} S_{k} \partial^{\mathbf{i}} \\
& =S_{k} D+p_{\hat{k}} D_{k}^{-} .
\end{aligned}
$$

This completes the proof.

By using this proposition, we can reduce a PDE for $n$ variables to a certain PDE for $n-1$ variables. For any $D=\sum_{\mathbf{i} \in \mathbf{Z}_{\geq 0}^{n}} b_{\mathbf{i}} \partial^{\mathbf{i}} \in \mathbf{C}\left[\partial_{1}, \cdots, \partial_{n}\right]$ and for any $k \in[1, n]$, we define $D_{\hat{k}} \in \mathbf{C}\left[\partial_{1}, \cdots, \partial_{k-1}, \partial_{k+1}, \cdots, \partial_{n}\right]$ by the formula

$$
D_{\hat{k}}=\sum_{\substack{\mathbf{i} \in \mathbf{Z}_{\geq 0}^{n} \\ i_{k}=0}} b_{\mathbf{i}} \partial^{\mathbf{i}} \in \mathbf{C}\left[\partial_{1}, \cdots, \partial_{k-1}, \partial_{k+1}, \cdots, \partial_{n}\right] .
$$

Proposition 5.3. Let $D \in \mathbf{C}\left[\partial_{1}, \cdots, \partial_{n}\right]$. Suppose that $f \in \mathbf{C}\left[w_{1}, \cdots\right.$, $\left.w_{n}\right]$ satisfies $D \cdot f=0$. Then a polynomial $g \in \mathbf{C}\left[w_{1}, \cdots, w_{k-1}, w_{k+1}, \cdots, w_{n}\right]$ satisfies $D \cdot\left(S_{k} f+g\right)=0$ if and only if $D_{\hat{k}} \cdot g=-p_{\hat{k}} D_{k}^{-} \cdot f$. 
Proof. By using Proposition 5.2, we can compute as follows.

$$
\begin{aligned}
D \cdot\left(S_{k} f+g\right) & =D S_{k} \cdot f+D_{\hat{k}} \cdot g \\
& =\left(S_{k} D+p_{\hat{k}} D_{k}^{-}\right) \cdot f+D_{\hat{k}} \cdot g \\
& =p_{\hat{k}} D_{k}^{-} \cdot f+D_{\hat{k}} \cdot g .
\end{aligned}
$$

This completes the proof.

For the two variables case, this proposition enables us to obtain a simple procedure which generates a complete system of solutions.

Proposition 5.4. Let $D \in \mathbf{C}\left[\partial_{1}, \partial_{2}\right]$. Suppose that $D_{\hat{k}}$ is a monomial for $k=1$ or 2 , say $D_{\hat{k}}=a \partial_{3-k}^{m}$, and let $O p_{k}=S_{k}-(1 / a) S_{3-k}^{m} p_{\hat{k}} D_{k}^{-}$. Then $O p_{k}\left(\operatorname{Sol}(D)_{N}\right) \subset \operatorname{Sol}(D)_{N+1}$ and $\operatorname{Op}_{k}\left(H \operatorname{Sol}(D)_{N}\right) \subset H \operatorname{Sol}(D)_{N+1}$ for any $N \geq 0$.

Proof. Since

$$
\begin{aligned}
D_{\hat{k}}\left(-(1 / a) S_{3-k}^{m} p_{\hat{k}} D_{k}^{-}\right) & =a \partial_{3-k}^{m}\left(-(1 / a) S_{3-k}^{m} p_{\hat{k}} D_{k}^{-}\right) \\
& =-\left(\partial_{3-k}^{m} S_{3-k}^{m}\right) p_{\hat{k}} D_{k}^{-}=-p_{\hat{k}} D_{k}^{-},
\end{aligned}
$$

it follows from Proposition 5.3 that $O p_{k}$ maps a solution to another solution. The assertions for degree are clear from the definition. This completes the proof.

We illustrate how this proposition works by the examples of PDE's treated in the previous section.

Example 5.5.1. Let $D_{\text {harmonic }}=\partial_{1}^{2}+\partial_{2}^{2}$ (see Example 4.7.1). The polynomial solutions of the Laplace equation are, of course, well known. Our aim here, however, is to verify the validity of our results through the known solutions. The polynomial $f=w_{1}$ is trivially a solution, so that we take this $f$ as in Proposition 5.3. Moreover since $D_{\hat{1}}=\partial_{2}^{2}$ and $D_{\hat{2}}=\partial_{1}^{2}$, we can employ Proposition 5.4 for $k=1,2$ with $a=1, m=2$. Since $D_{1}^{-}=\partial_{1}, D_{2}^{-}=\partial_{2}$, we have

$$
O p_{1} \cdot f=\left(S_{1}-S_{2}^{2} p_{\hat{1}} \partial_{1}\right) \cdot w_{1}=S_{1} \cdot w_{1}-S_{2}^{2} \cdot 1=w_{1}^{2} / 2-w_{2}^{2} / 2,
$$

and

$$
O p_{2} \cdot f=\left(S_{2}-S_{1}^{2} p_{\hat{2}} \partial_{2}\right) \cdot w_{1}=S_{2} \cdot w_{1}=w_{1} w_{2} .
$$

These two solutions constitute a basis of the space $\mathrm{HSol}\left(D_{\text {harmonic }}\right)_{2}$. Generally we can show that $\left(O p_{1}\right)^{N} \cdot 1$ and $\left(O p_{1}\right)^{N-1} O p_{2} \cdot 1$ provide us with a basis of 
$H \operatorname{Sol}\left(D_{\text {harmonic }}\right)_{N}$. For notational simplicity, we let the word $\left(\varepsilon_{1} \varepsilon_{2} \cdots \varepsilon_{N}\right)$ denote the composite $O p_{\varepsilon_{1}} O p_{\varepsilon_{2}} \cdots O p_{\varepsilon_{N}} \in \operatorname{End}_{\mathbf{C}}\left(P_{2}\right)$ for any $\left(\varepsilon_{1}, \varepsilon_{2}, \cdots, \varepsilon_{N}\right) \in$ $\{1,2\}^{N}$. Then one can show by induction that

$$
\begin{aligned}
\left(1^{N}\right) \cdot 1 & =(\text { const }) \sum_{k \in \mathbf{Z}}(-1)^{k}\left(\begin{array}{c}
N \\
2 k
\end{array}\right) w_{1}^{N-2 k} w_{2}^{2 k} \\
\left(1^{N-1} 2\right) \cdot 1 & =(\text { const }) \sum_{k \in \mathbf{Z}}(-1)^{k}\left(\begin{array}{c}
N \\
2 k+1
\end{array}\right) w_{1}^{N-2 k-1} w_{2}^{2 k+1} .
\end{aligned}
$$

These are apparently linearly independent. Since we have already seen in Example 4.7.1 that $\operatorname{dim} H \operatorname{Sol}\left(D_{\text {harmonic }}\right)_{N}=2$ for any $N \geq 1$, we have $H \operatorname{Sol}\left(D_{\text {harmonic }}\right)_{N}=\left\langle\left(1^{N}\right) \cdot 1,\left(1^{N-1} 2\right) \cdot 1\right\rangle_{\mathbf{C}}$ for any $N \geq 1$.

Example 5.5.2. $\quad D_{\text {biharmonic }}=\partial_{1}^{4}+2 \partial_{1}^{2} \partial_{2}^{2}+\partial_{2}^{4}$ (see Example 4.7.2). Since $D_{\hat{1}}=\partial_{2}^{4}, D_{\hat{2}}=\partial_{1}^{4}$, we can employ Proposition 5.4 for both $k=1,2$ with $a=1, m=4$. By definition, we have $D_{1}^{-}=\partial_{1}^{3}+2 \partial_{1} \partial_{2}^{2}, D_{2}^{-}=\partial_{2}^{3}+2 \partial_{1}^{2} \partial_{2}$. Taking $f=w_{1}^{3}$ as a starting solution, for example, we have

$$
\begin{aligned}
& O p_{1} \cdot f=\left(S_{1}-S_{2}^{4} p_{\hat{1}} D_{1}^{-}\right) \cdot w_{1}^{3}=S_{1} \cdot w_{1}^{3}-S_{2}^{4} \cdot 6=w_{1}^{4} / 4-w_{2}^{4} / 4, \\
& O p_{2} \cdot f=\left(S_{2}-S_{1}^{4} p_{\hat{2}} D_{2}^{-}\right) \cdot w_{1}^{3}=S_{2} \cdot w_{1}^{3}=w_{1}^{3} w_{2} .
\end{aligned}
$$

In general, one can check by induction that

$$
\begin{aligned}
\left(1^{N}\right) \cdot 1 & =(\text { const }) \sum_{k \in \mathbf{Z}}(-1)^{k} k\left(\begin{array}{c}
N \\
2 k+2
\end{array}\right) w_{1}^{N-(2 k+2)} w_{2}^{2 k+2}, \\
\left(1^{N-1} 2\right) \cdot 1 & =(\text { const }) \sum_{k \in \mathbf{Z}}(-1)^{k} k\left(\begin{array}{c}
N \\
2 k+3
\end{array}\right) w_{1}^{N-(2 k+3)} w_{2}^{2 k+3}, \\
\left(1^{N-2} 2^{2}\right) \cdot 1 & =(\text { const }) \sum_{k \in \mathbf{Z}}(-1)^{k} k\left(\begin{array}{c}
N \\
2 k
\end{array}\right) w_{1}^{N-2 k} w_{2}^{2 k}, \\
\left(1^{N-3} 2^{3}\right) \cdot 1 & =(\text { const }) \sum_{k \in \mathbf{Z}}(-1)^{k} k\left(\begin{array}{c}
N \\
2 k+1
\end{array}\right) w_{1}^{N-(2 k+1)} w_{2}^{2 k+1} .
\end{aligned}
$$

Since these are evidently linearly independent and $\operatorname{dim} H \operatorname{Sol}\left(D_{\text {biharmonic }}\right)_{N}=$ 4 for $N \geq 3$, as is seen in Example 4.7.2, we see that

$$
H \operatorname{Sol}(D)_{N}=\left\langle\left(1^{N}\right) \cdot 1,\left(1^{N-1} 2\right) \cdot 1,\left(1^{N-2} 2^{2}\right) \cdot 1,\left(1^{N-3} 2^{3}\right) \cdot 1\right\rangle_{\mathbf{C}}, \text { for } N \geq 3
$$

Example 5.5.3. $\quad D_{\text {harmonic }}=\partial_{1}^{2}+\partial_{2}^{2}-\partial_{1}^{2} \partial_{2}-\partial_{1} \partial_{2}^{2}$ (see Example 4.7.4). Let $D=D_{\text {harmonic }}$. Since $D_{\hat{1}}=\partial_{2}^{2}, D_{\hat{2}}=\partial_{1}^{2}$, we can employ Proposition 
5.4 for both $k$ with $a=1, m=2$. By definition, we have $D_{1}^{-}=\partial_{1}-\partial_{1} \partial_{2}-$ $\partial_{2}^{2}, D_{2}^{-}=\partial_{2}-\partial_{1}^{2}-\partial_{1} \partial_{2}$. Therefore we have the following list (up to constant):

$$
\begin{array}{ll}
(1) \cdot 1=w_{1}, & (2) \cdot 1=w_{2} \\
\left(1^{2}\right) \cdot 1=w_{1}^{2}-w_{2}^{2}, & (12) \cdot 1=w_{1} w_{2}, \\
\left(1^{3}\right) \cdot 1=w_{1}^{3}-3 w_{1} w_{2}^{2}-3 w_{2}^{2}, & \left(1^{2} 2\right) \cdot 1=3 w_{1}^{2} w_{2}-w_{2}^{3}+3 w_{2}^{2},
\end{array}
$$

and so on. If we denote the degree- $N$-part of a polynomial $f$ by $(f)_{N}$, then one can show by induction that

$$
\begin{aligned}
\left(\left(1^{N}\right) \cdot 1\right)_{N} & =(\text { const }) \sum_{0 \leq k \leq N / 2}(-1)^{k}\left(\begin{array}{l}
N \\
2 k
\end{array}\right) w_{1}^{N-2 k} w_{2}^{2 k}, \\
\left(\left(1^{N-1} 2\right) \cdot 1\right)_{N} & =(\text { const }) \sum_{0 \leq k \leq(N-1) / 2}(-1)^{k}\left(\begin{array}{c}
N \\
2 k+1
\end{array}\right) w_{1}^{N-(2 k+1)} w_{2}^{2 k+1} .
\end{aligned}
$$

Since these are linearly independent, we see that

$\operatorname{Sol}\left(D_{\text {harmonic }}\right)_{\leq N}=\mathbf{C} \cdot 1 \oplus\left\langle\left(1^{k}\right) \cdot 1,\left(1^{k-1} 2\right) \cdot 1 ; 1 \leq k \leq N\right\rangle_{\mathbf{C}}$ for any $N \geq 1$.

Furthermore we see that $\left(1^{3}\right) \cdot 1+\left(1^{2} 2\right) \cdot 1=w_{1}^{3}+3 w_{1}^{2} w_{2}-3 w_{1} w_{2}^{2}-w_{2}^{3}$ is homogeneous of degree three, and one can show that

$$
\begin{aligned}
& H \operatorname{Sol}(D)_{0}=\langle 1\rangle_{\mathbf{C}}, H \operatorname{Sol}(D)_{1}=\langle(1) \cdot 1,(2) \cdot 1\rangle_{\mathbf{C}}, \\
& H \operatorname{Sol}(D)_{2}=\left\langle\left(1^{2}\right) \cdot 1,(12) \cdot 1\right\rangle_{\mathbf{C}}, H \operatorname{Sol}(D)_{3}=\left\langle\left(1^{3}\right) \cdot 1+\left(1^{2} 2\right) \cdot 1\right\rangle_{\mathbf{C}} .
\end{aligned}
$$

There are no homogeneous solutions of degree $N$ for $N \geq 4$, as is shown in Example 4.8.1.

\section{§6. Discrete Tomography}

In this section we translate the results on the space of polynomial solutions of PDE obtained in the previous sections into those on discrete tomography and examine our results by several examples of windows.

Now that we have solved the problem in the step (C) of Algorithm 3.3, we can apply the whole machinery in the paper to find zero-sum arrays of polynomial growth for any windows. We illustrate the process by several examples of windows related to the PDE's treated in the previous sections. 


\section{$\S$ 6.1. Window $t_{\text {corner }}^{n}$}

The window $\mathbf{t}_{\text {corner }}^{n}$ is defined by

$$
\left(\mathbf{t}_{\text {corner }}^{n}\right)_{\mathbf{i}}= \begin{cases}1, & \text { if } \mathbf{i}=\mathbf{e}_{k} \text { for some } k \\ -n, & \text { if } \mathbf{i}=\mathbf{0}, \\ 0, & \text { otherwise. }\end{cases}
$$

The characteristic polynomial $m_{\mathbf{t}_{\text {corner }}^{n}}$ is given by $m_{\mathbf{t}_{\text {corner }}^{n}}(\mathbf{z})=\sum_{1 \leq k \leq n} z_{k}-$ $n$, and its zero locus on $\mathbf{T}^{n}$ is $V_{\mathbf{T}^{n}}\left(m_{\mathbf{t}_{\text {corner }}^{n}}\right)=\{(1,1, \cdots, 1)\}$, since $\left|z_{k}\right|=$ $1,1 \leq k \leq n$. As a local coordinate at $\mathbf{1}=(1,1, \cdots, 1) \in \mathbf{T}^{n}$, we take $w_{k}=z_{k}-1,1 \leq k \leq n$. Then the translated characteristic polynomial $m_{\mathbf{t}_{\text {corner }}^{n}}^{\mathbf{1}}$ is expressed as $m_{\mathbf{t}_{\text {corner }}^{n}}^{\mathbf{1}}(\mathbf{w})=\sum_{1 \leq k \leq n} w_{k}$. Since $F_{-}\left(\sum_{1 \leq k \leq n} w_{k}\right)=$ $-\sum_{1 \leq k \leq n} \partial_{k}$ by the definition, we are led to deal with $\operatorname{Sol}\left(-\sum_{1 \leq k \leq n} \partial_{k}\right)_{\leq N}=$ $\operatorname{Sol}\left(\sum_{1 \leq k \leq n} \partial_{k}\right)_{\leq N}$. The dimension formula of the space of the polynomial solutions is deduced already in Example 4.7.3. Therefore we are to find actual solutions. Let $D=D_{\text {corner }}$. Let $\mu_{n}$ denote the set of $n$-th roots of unity, and for any $\zeta \in \mu_{n}-\{1\}$, let $w_{\zeta}=\sum_{1 \leq k \leq n} \zeta^{k-1} w_{k}$. Then we see that $D w_{\zeta}=$ $\sum_{1 \leq k \leq n} \zeta^{k-1}\left(\partial_{1}+\cdots+\partial_{n}\right) w_{k}=\sum_{1 \leq k \leq n} \zeta^{k-1}=0$. Therefore any products of $w_{\zeta}, \zeta \in \mu_{n}-\{1\}$, belong to $\operatorname{Sol}(D)$. Furthermore if we put $w(i)=w_{\zeta_{n}^{i}}, 1 \leq$ $i \leq n-1$, with a fixed primitive $n$-th root of unity $\zeta_{n}$, then they are linearly independent, and hence the products $w\left(i_{1}\right) w\left(i_{2}\right) \cdots w\left(i_{N}\right), \quad 1 \leq i_{1} \leq i_{2} \leq$ $\cdots \leq i_{N} \leq n-1$, for fixed $N$ are linearly independent too. Note that the number of them is equal to $\left(\begin{array}{c}n-2+N \\ n-2\end{array}\right)$, which coincides with the dimension of $H \operatorname{Sol}(D)_{N}$, as is seen in Example 4.7.3. Therefore

$$
H \operatorname{Sol}(D)_{N}=\left\langle w\left(i_{1}\right) w\left(i_{2}\right) \cdots w\left(i_{N}\right) ; 1 \leq i_{1} \leq i_{2} \leq \cdots \leq i_{N} \leq n-1\right\rangle_{\mathbf{C}} .
$$

In order to go to Step (D) in Algorithm 3.3, we put $\partial(i)=\sum_{1 \leq k \leq n} \zeta_{n}^{i(k-1)} \partial_{k}$ so that $F_{-}(\partial(i))=w(i)$. Then Corollary 3.1.1 together with (6.1) implies the following.

Proposition 6.1.1. Notation being as above, we have

$$
\begin{aligned}
& \operatorname{Diff}\left(m_{\mathbf{t}_{\text {corner }}^{n}}\right) \\
& \quad=\mathbf{C} \cdot 1 \oplus \bigoplus_{N \geq 1}\left\langle\partial\left(i_{1}\right) \partial\left(i_{2}\right) \cdots \partial\left(i_{N}\right) ; 1 \leq i_{1} \leq i_{2} \leq \cdots \leq i_{N} \leq n-1\right\rangle_{\mathbf{C}} .
\end{aligned}
$$

Translating this result by the algorithm, we can determine completely the space of zero-sum arrays for the window $\mathbf{t}_{\text {corner }}^{n}$. We illustrate the process of translation through the two-dimensional case. In this case 
Proposition 6.1.1 says that the degree $N$ part of $\operatorname{Diff}\left(m_{\mathbf{t}_{\text {corner }}^{n}}\right)$ is a onedimensional vector space generated by $\partial(1)^{N}=\left(\partial_{w_{1}}+\zeta_{2} \partial_{w_{2}}\right)^{N}=$ $\left(\partial_{w_{1}}-\partial_{w_{2}}\right)^{N}=\sum_{0 \leq j \leq N}\left(\begin{array}{c}N \\ j\end{array}\right)(-1)^{j} \partial_{w_{1}}^{N-j} \partial_{w_{2}}^{j}$. Accordingly let $f_{N}(x, y)=$ $\sum_{0 \leq j \leq N}\left(\begin{array}{c}N \\ j\end{array}\right)(-1)^{j} x^{\overline{N-j}} y^{\bar{j}}$ and let $g_{N}(x, y)=(-1)^{N} f_{N}(-x,-y)$. (The coefficient $(-1)^{N}$ is inessential and attached in order to make the coefficient of $x^{N}$ positive.) Then it follows from Theorem 3.2 that we have

$$
\mathbf{A}_{\mathbf{t}_{\text {corner }}^{n}}^{N}=\mathbf{C} \cdot \mathbf{1} \oplus \bigoplus_{N \geq 1}\left\langle\left(g_{N}\left(k_{1}, k_{2}\right)\right)_{\left.\left(k_{1}, k_{2}\right) \in \mathbf{Z}^{2}\right\rangle_{\mathbf{C}}} .\right.
$$

For example, when $N=1,2,3$, we have

$$
\begin{aligned}
g_{1}(x, y) & =(x-1)-(y-1)=x-y, \\
g_{2}(x, y) & =(x-1)(x-2)-2(x-1)(y-1)+(y-1)(y-2) \\
& =(x-y)^{2}-(x+y)+2, \\
g_{3}(x, y) & =(x-y)^{3}-3\left(x^{2}-y^{2}\right)+8(x-y) .
\end{aligned}
$$

The corresponding arrays are depicted as follows:

$$
\begin{array}{ccccc}
g_{1}: & & & & \\
-3 & -2 & -1 & 0 & 1 \\
-2 & -1 & 0 & 1 & 2 \\
1 & 0 & 1 & 2 & 3 \\
0 & 1 & 2 & 3 & 4 \\
g_{3}: & & & & \\
-24 & 0 & 6 & 0 & -12 \\
-12 & 0 & 0 & -6 & -12 \\
-6 & 0 & 0 & 0 & 6 \\
-0 & 6 & 12 & 24 & 48
\end{array}
$$$$
g_{2}:
$$$$
\begin{array}{lllll}
8 & 2 & -2 & -4 & -4
\end{array}
$$$$
\begin{array}{lllll}
4 & 0 & -2 & -2 & 0
\end{array}
$$

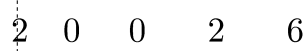$$
2 \cdots 2 \cdots 4-8 \cdots 14 \cdots
$$

One can check by inspection that these are actually zero-sum arrays for $\mathbf{t}_{\text {corner }}^{n}$.

\section{$\S 6.2 . \quad$ Window $t_{l e g}$}

This is a two-dimensional window defined by

$$
\left(\mathbf{t}_{\text {leg }}\right)_{\mathbf{i}}=\left\{\begin{array}{lll}
1, & \text { if } \quad \mathbf{i}=2 \mathbf{e}_{k} & \text { for } k=1 \quad \text { or } \quad 2, \\
-2, & \text { if } \mathbf{i}=\mathbf{e}_{k} & \text { for } k=1 \quad \text { or } \quad 2 \\
2, & \text { if } \quad \mathbf{i}=\mathbf{0}, & \\
0, & \text { otherwise }
\end{array}\right.
$$


Its characteristic polynomial and the zero locus are given by

$$
\begin{aligned}
m_{\mathbf{t}_{\text {leg }}}(\mathbf{z}) & =\left(z_{1}-1\right)^{2}+\left(z_{2}-1\right)^{2} . \\
V_{\mathbf{T}^{2}}\left(m_{\mathbf{t}_{\text {leg }}}\right) & =\{(1,1),(i,-i),(-i, i)\} .
\end{aligned}
$$

First we deal with the space $\operatorname{Diff}\left(m_{\mathbf{t}_{l e g}}^{(1,1)}\right)$. As a local coordinate at $(1,1)$, we take $w_{k}=z_{k}-1, k=1,2$. Then the translated characteristic polynomial is expressed as $m_{\mathbf{t}_{l e g}}^{(1,1)}\left(w_{1}, w_{2}\right)=w_{1}^{2}+w_{2}^{2}$. Since $F_{-}\left(w_{1}^{2}+w_{2}^{2}\right)=\partial_{w_{1}}^{2}+\partial_{w_{2}}^{2}$, we are to determine $\operatorname{Sol}\left(\partial_{w_{1}}^{2}+\partial_{w_{2}}^{2}\right)$. This, however, is already considered in Example 5.5.1, and we know that the degree- $N$ part of Diff $\left(m_{\mathbf{t}_{l e g}}^{(1,1)}\right)$ for $N \geq 1$ is a two-dimensional vector space generated by $\sum_{k \in \mathbf{Z}}(-1)^{k}\left(\begin{array}{c}N \\ 2 k\end{array}\right) \partial_{w_{1}}^{N-2 k} \partial_{w_{2}}^{2 k}$ and $\sum_{k \in \mathbf{Z}}(-1)^{k}\left(\begin{array}{c}N \\ 2 k+1\end{array}\right) \partial_{w_{1}}^{N-2 k-1} \partial_{w_{2}}^{2 k+1}$. Accordingly we let

$$
\begin{aligned}
p_{N}(x, y) & =\sum_{0 \leq k \leq N / 2}(-1)^{k}\left(\begin{array}{l}
N \\
2 k
\end{array}\right) x^{\overline{N-2 k}} y^{\overline{2 k}}, \\
q_{N}(x, y) & =\sum_{0 \leq k<N / 2}(-1)^{k}\left(\begin{array}{c}
N \\
2 k+1
\end{array}\right) x^{\overline{N-2 k-1}} y^{\overline{2 k+1}},
\end{aligned}
$$

and put $p_{N}^{-}(x, y)=p_{N}(-x,-y), q_{N}^{-}(x, y)=q_{N}(-x,-y)$. Then we have

$$
\mathbf{A}_{\mathbf{t}_{l e g}}^{N}=\mathbf{C} \cdot \mathbf{1} \oplus \bigoplus_{N \geq 1}\left\langle\left(p_{N}^{-}\left(k_{1}, k_{2}\right)\right)_{\left(k_{1}, k_{2}\right) \in \mathbf{Z}^{2}},\left(q_{N}^{-}\left(k_{1}, k_{2}\right)\right)_{\left(k_{1}, k_{2}\right) \in \mathbf{Z}^{2}}\right\rangle_{\mathbf{C}} .
$$

For small values of $N$, these basis are illustrated below:

$$
\begin{array}{lll}
N & p_{N}^{-}(x, y) & q_{N}^{-}(x, y) \\
1 & x-1 & y-1 \\
2 & x^{2}-y^{2}-3(x-y) & 2 x y-2(x+y)+2 \\
3 & x^{3}-3 x y^{2}-6 x^{2}+9 x y+3 y^{2}+5 x-9 y & 3 x^{2} y-y^{3}-3 x^{2}-9 x y+6 y^{2}+9 x-5 y
\end{array}
$$

The arrays corresponding to these are depicted as follows:

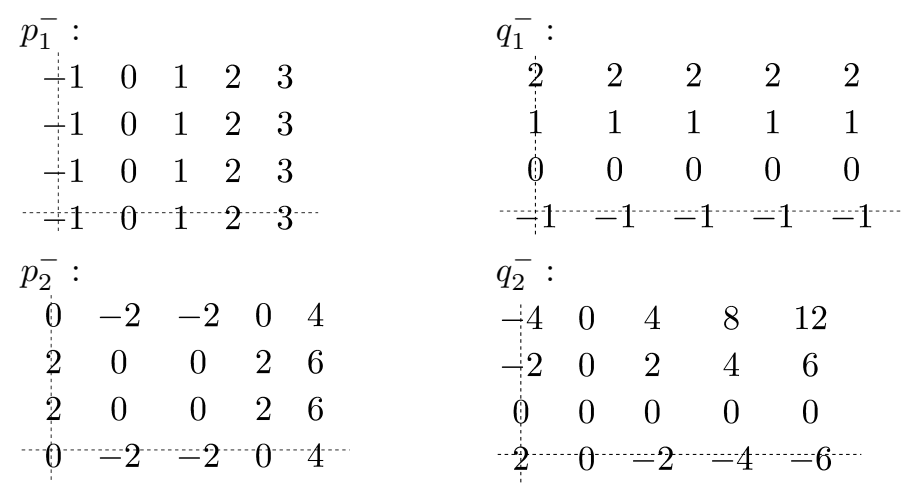


Next we deal with the space Diff $\left(m_{\mathbf{t}_{\text {leg }}}^{(i,-i)}\right)$. As a local coordinate at $(i,-i)$, we take $w_{1}=z_{1}-i, w_{2}=z_{2}+i$. Then the translated characteristic polynomial is expressed as

$m_{\mathbf{t}_{\text {leg }}}^{(i,-i)}\left(w_{1}, w_{2}\right)=\left(w_{1}+i-1\right)^{2}+\left(w_{2}-i-1\right)^{2}=w_{1}^{2}+w_{2}^{2}+2(i-1) w_{1}-2(i+1) w_{2}$.

Therefore we have $F_{-}\left(m_{\mathbf{t}_{l e g}}^{(i,-i)}\right)=\partial_{1}^{2}+\partial_{2}^{2}-2(i-1) \partial_{1}+2(i+1) \partial_{2}$. Let $D$ denote this $\partial$-polynomial. One notices that neither $D_{\hat{1}}$ nor $D_{\hat{2}}$ is a monomial, and hence Proposition 5.4 is not of use as it is. But by an appropriate coordinate transformation, we can change $D$ into a form for which the proposition is applicable as follows. Let $v_{1}=w_{1}+i w_{2}, v_{2}=w_{1}-i w_{2}$. Then we have

$$
\partial_{1}=\partial_{w_{1}}=\partial_{v_{1}}+\partial_{v_{2}}, \partial_{2}=\partial_{w_{2}}=i \partial_{v_{1}}-i \partial_{v_{2}} .
$$

Therefore in the coordinate system $\left(v_{1}, v_{2}\right)$, our differential operator $D$ is expressed as

$$
D=4\left(\partial_{v_{1}} \partial_{v_{2}}+(1-i) \partial_{v_{2}}\right) .
$$

Since mdeg $D=1$, it follows from Theorem 4.3 that

$$
\operatorname{dim} \operatorname{Sol}(D)_{\leq N}=\left(\begin{array}{c}
2+N \\
2
\end{array}\right)-\left(\begin{array}{c}
1+N \\
2
\end{array}\right)=N+1 .
$$

Furthermore, since $D_{\hat{1}}=4(1-i) \partial_{v_{2}}$ is a monomial, we can apply Proposition 5.4 with $k=1, a=4(1-i)$, and $m=1$. Noticing that $D_{1}^{-}=4 \partial_{v_{2}}$, we obtain that $\left(1^{n}\right) \cdot 1=($ const. $) v_{1}^{n}$, and hence we have

$$
\operatorname{Sol}(D)_{\leq N}=\left\langle\left(1^{n}\right) \cdot 1 ; n \in[0, N]\right\rangle_{\mathbf{C}} .
$$

In particular, we see that $H \operatorname{Sol}(D)_{N}=\left\langle v_{1}^{N}\right\rangle_{\mathbf{C}}=\left\langle\left(w_{1}+i w_{2}\right)^{N}\right\rangle_{\mathbf{C}}$.

Remark 6.2.1. This gives us an example of $\partial$-polynomial $D$ such that $H \operatorname{Sol}(D)_{N} \neq\{0\}$ for any $N$. Indeed we have $D_{2}=4 \partial_{v_{1}} \partial_{v_{2}}, D_{1}=4(1-$ i) $\partial_{v_{2}}$ in the notation of Theorem 4.8, and we see that $V\left(F_{+}\left(D_{2}\right), F_{+}\left(D_{1}\right)\right)=$ $V\left(4 v_{1} v_{2}, 4(1-i) v_{2}\right)=V\left(v_{2}\right)$ is a line in $\mathbf{A}^{2}$. Hence Theorem 4.8 too implies that $H \operatorname{Sol}(D)_{N} \neq\{0\}$ for any $N$.

Next we will find the zero-sum arrays corresponding to the solutions of low degree given above. Note that $(-\mathbf{k})^{\mathbf{s}} \mathbf{p}^{\mathbf{k}-\mathbf{s}}=i^{k_{1}}(-i)^{k_{2}}=(-1)^{k_{2}} i^{k_{1}+k_{2}}$ for $\mathbf{k}=\left(k_{1}, k_{2}\right), \mathbf{s}=(0,0), \mathbf{p}=(i,-i)$. Therefore it follows from Theorem 2.6 that $\left((-1)^{k_{2}} i^{k_{1}+k_{2}}\right)_{\left(k_{1}, k_{2}\right) \in \mathbf{Z}^{2}} \in \mathbf{A}_{\mathbf{t}_{\text {leg }}}^{0}$. We call this array $\mathbf{a}_{0}$. This array is depicted 
as

$$
\begin{array}{ccccc}
\mathbf{a}_{0} ; & & & & \\
i & -1 & -i & 1 & i \\
1 & -i & 1 & i & -1 \\
i & 1 & i & -1 & -i
\end{array}
$$

and one can check directly that this is a zero-sum array for $\mathbf{t}_{l e g}$. Furthermore we see that $(-\mathbf{k})^{\overline{\mathbf{s}}} \mathbf{p}^{\mathbf{k}-\mathbf{s}}=\left(-k_{1}+1\right) i^{k_{1}-1}(-i)^{k_{2}}$ for $\mathbf{k}=\left(k_{1}, k_{2}\right), \mathbf{s}=(1,0), \mathbf{p}=$ $(i,-i)$, and $(-\mathbf{k})^{\overline{\mathbf{s}}} \mathbf{p}^{\mathbf{k}-\mathbf{s}}=\left(-k_{2}+1\right) i^{k_{1}}(-i)^{k_{2}-1}$ for $\mathbf{k}=\left(k_{1}, k_{2}\right), \mathbf{s}=(0,1), \mathbf{p}=$ $(i,-i)$. Hence we see that

$$
\begin{aligned}
G_{(i,-i)}\left(F_{-}^{-1}\left(w_{1}+i w_{2}\right)\right) & =G_{(i,-i)}\left(\partial_{1}+i \partial_{2}\right) \\
& =\left(-k_{1}+1\right) i^{k_{1}-1}(-i)^{k_{2}}+i\left(-k_{2}+1\right) i^{k_{1}}(-i)^{k_{2}-1} \\
& =i^{k_{1}+k_{2}-1}(-1)^{k_{2}-1}\left(\left(k_{1}-1\right)-i\left(k_{2}-1\right)\right)
\end{aligned}
$$

Subtracting an appropriate constant multiple of the basis element $\left((-1)^{k_{2}} i^{k_{1}+k_{2}}\right)_{\left(k_{1}, k_{2}\right) \in \mathbf{Z}^{2}}$ of $\mathbf{A}_{\mathbf{t}_{l e g}}^{0}$ obtained above, we see that $\left(i^{k_{1}+k_{2}}(-1)^{k_{2}}\left(k_{1}-\right.\right.$ $\left.\left.i k_{2}\right)\right)_{\left(k_{1}, k_{2}\right) \in \mathbf{Z}^{2}} \in \mathbf{A}_{\mathbf{t}_{l e g}}^{1}$. We call this array $\mathbf{a}_{1}$. It is depicted as

$$
\begin{array}{ccccc}
\mathbf{a}_{1}: & & & & \\
3 & -1+3 i & -3-2 i & 3-3 i & 3+4 i \\
2 i & -2-i & 2-2 i & 2+3 i & -4+2 i \\
1 & 1-i & 1+2 i & -3+i & -1-4 i \\
\hdashline & i & -2 & -3 i & 4
\end{array}
$$

It remains to deal with the space Diff $\left(m_{\mathbf{t}_{l e g}}^{(-i, i)}\right)$. This is, however, reduced to the previous case, since it is isomorphic to $\operatorname{Diff}\left(m_{\mathbf{t}_{l e g}}^{(i,-i)}\right)$ via complex conjugation. Hence we see that $\overline{\mathbf{a}}_{0}=\left((-1)^{k_{2}}(-i)^{k_{1}+k_{2}}\right)_{\left(k_{1}, k_{2}\right) \in \mathbf{Z}^{2}}$ gives rise to a basis element of $\mathbf{A}_{\mathbf{t}_{\text {leg }}}^{0}$, and $\overline{\mathbf{a}}_{1}=\left((-i)^{k_{1}+k_{2}}(-1)^{k_{2}}\left(k_{1}+i k_{2}\right)\right)_{\left(k_{1}, k_{2}\right) \in \mathbf{Z}^{2}}$ of $\mathbf{A}_{\mathbf{t}_{\text {leg }}}^{1}$. They are illustrated as follows:

$\overline{\mathbf{a}}_{0}:$

$$
\begin{array}{r:cccc}
-i & -1 & i & 1 & -i \\
-1 & i & 1 & -i & -1 \\
i & 1 & -i & -1 & i \\
1 & -i & -1 & i & 1
\end{array}
$$

$\overline{\mathbf{a}}_{1}:$

$$
\begin{array}{ccccc}
3 & -1-3 i & -3+2 i & 3+3 i & 3-4 i \\
2 i & -2+i & 2+2 i & 2-3 i & -4-2 i \\
1 & 1+i & 1-2 i & -3-i & -1+4 i \\
\hdashline & -i & -2 & 3 i & -4
\end{array}
$$

Furthermore, by adding and subtracting the pairs of the conjugate arrays, we 
obtain $\mathbf{Z}$-valued arrays as follows:

$$
\begin{aligned}
& \left(\mathbf{a}_{0}+\overline{\mathbf{a}}_{0}\right) / 2 \text { : } \\
& \begin{array}{lllll}
0 & -1 & 0 & 1 & 0
\end{array} \\
& \begin{array}{rrrrr}
1 & 0 & 1 & 0 & -1
\end{array} \\
& \begin{array}{lllll}
0 & 1 & 0 & -1 & 0
\end{array} \\
& 1-\theta-1-\theta-1 \cdots \\
& \left(\mathbf{a}_{1}+\overline{\mathbf{a}}_{1}\right) / 2 \text { : } \\
& \begin{array}{lllll}
3 & -1 & -3 & 3 & 3
\end{array} \\
& \begin{array}{lllll}
0 & -2 & 2 & 2 & -4
\end{array} \\
& \begin{array}{lllll}
-1 & 1 & 1 & -3 & -1
\end{array} \\
& \theta \quad \theta \quad-2 \cdots \quad 0 \quad-\quad-4
\end{aligned}
$$

$$
\begin{array}{ccccc}
\left(\mathbf{a}_{0}-\overline{\mathbf{a}}_{0}\right) /(2 i): & & \\
1 & 0 & -1 & 0 & 1 \\
0 & -1 & 0 & 1 & 0 \\
1 & 0 & 1 & 0 & -1 \\
0 & 1 & 0 & -1 & \theta \\
\left(\mathbf{a}_{1}-\overline{\mathbf{a}}_{1}\right) /(2 i): & & \\
0 & 3 & -2 & -3 & 4 \\
2 & -1 & -2 & 3 & 2 \\
0 & -1 & 2 & 1 & -4 \\
0 & 1 & 0 & -3 & -
\end{array}
$$

\section{§6.3. Window $t_{\text {harmonic }}$}

The space $\mathbf{A}_{\mathbf{t}}^{0}$ is investigated already in our previous paper [3, 4]. Our results in the present paper, however, enable us to determine the structure of the space $\mathbf{A}_{\mathbf{t}_{h a}}^{N}$ for any $N$. Recall that the window is defined by

$$
\left(\mathbf{t}_{\text {harmonic }}\right)_{\mathbf{i}}= \begin{cases}1, & \text { if } \mathbf{i}=(1,0),(0,1),(2,1),(1,2), \\ -4, & \text { if } \mathbf{i}=(1,1) \\ 0, & \text { otherwise }\end{cases}
$$

This window coincides with the one in [3] up to translation. This causes no harm, since the space of zero-sum arrays does not change under translation of the window. Its characteristic polynomial is given by

$m_{\mathbf{t}_{\text {harmonic }}}(\mathbf{z})=z_{1}+z_{2}+z_{1}^{2} z_{2}+z_{1} z_{2}^{2}-4 z_{1} z_{2}=z_{1} z_{2}\left(z_{1}+1 / z_{1}+z_{2}+1 / z_{2}-4\right)$,

and hence $V_{\mathbf{T}^{2}}\left(m_{\mathbf{t}_{\text {harmonic }}}\right)=\{(1,1)\}$. If we put $w_{k}=z_{k}-1,1 \leq k \leq 2$, then the translated characteristic polynomial is expressed as $m_{\mathbf{t}_{\text {harmonic }}}^{(1,1)}\left(w_{1}, w_{2}\right)=$ $w_{1}^{2}\left(w_{2}+1\right)+\left(w_{1}+1\right) w_{2}^{2}$, and hence $F_{-}\left(m_{\mathbf{t}_{\text {harmonic }}}^{(1,1)}\right)=\partial_{w_{1}}^{2}+\partial_{w_{2}}^{2}-\partial_{w_{1}}^{2} \partial_{w_{2}}-$ $\partial_{w_{1}} \partial_{w_{2}}^{2}$. Therefore we are to determine $\operatorname{Sol}\left(\partial_{w_{1}}^{2}+\partial_{w_{2}}^{2}-\partial_{w_{1}}^{2} \partial_{w_{2}}-\partial_{w_{1}} \partial_{w_{2}}^{2}\right)$. This, however, is already considered in Example 5.5.3. Furthermore, since the window $\mathbf{t}_{\text {harmonic }}$ is symmetric with respect to its center, we have $\mathbf{A}_{\mathbf{t}_{\text {harmonic }}}^{N}=$ $\mathbf{A}_{\mathbf{t}_{\text {harmonic }}^{*}}^{N}$. Hence the solutions give us directly the zero-sum arrays for $\mathbf{t}_{\text {harmonic }}$ For $N=1,2,3$, the zero-sum arrays corresponding to these are depicted as fol- 
lows:

(1)

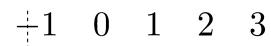

$\begin{array}{rllll}-1 & 0 & 1 & 2 & 3\end{array}$

$\begin{array}{rrrrr}-1 & 0 & 1 & 2 & 3\end{array}$

$1-\theta-1-2-3$
-2

$\left(1^{2}\right)$

$\begin{array}{lllll}0 & -2 & -2 & 0 & 4\end{array}$

$\begin{array}{lllll}2 & 0 & 0 & 2 & 6\end{array}$

$\begin{array}{lllll}2 & 0 & 0 & 2 & 6\end{array}$

$\theta \cdots-2 \cdots-2 \cdots \theta-4$

$\left(1^{3}\right)$

$114 \quad-156 \quad-180 \quad-180 \quad-150$

$\begin{array}{llllll}-66 & -84 & -84 & -60 & -6\end{array}$

$\begin{array}{lllll}-30 & -30 & -12 & 30 & 102\end{array}$

$\begin{array}{llll}6 & 6 & 36 & 90\end{array}$
(2)

\begin{tabular}{ccccc}
2 & 2 & 2 & 2 & 2 \\
1 & 1 & 1 & 1 & 1 \\
0 & 0 & 0 & 0 & 0 \\
\hdashline 1 & -1 & -1 & -1 & -1
\end{tabular}

$\begin{array}{rllll}-2 & 0 & 2 & 4 & 6\end{array}$

$\begin{array}{rrrrr}1 & 0 & 1 & 2 & 3\end{array}$

$\begin{array}{lllll}0 & 0 & 0 & 0 & 0\end{array}$

$1-0-1-2-3-2$

$\left(1^{2} 2\right)$

$\begin{array}{rrrrr}36 & 12 & 84 & 480 & 300\end{array}$

$6 \quad 30 \quad 84 \quad 156 \quad 246$

$\begin{array}{llllll}6 & 30 & 66 & 114 & 174\end{array}$

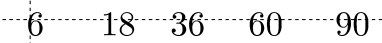

\section{References}

[1] R. E. Edwards, Fourier series. Vol. 2, Second edition, Springer, New York, 1982.

[2] R. L. Graham, D. E. Knuth and O. Patashnik, Concrete mathematics, Second edition, Addison-Wesley, Reading, MA, 1994.

[3] F. Hazama, Discrete tomography and the Hodge conjecture for certain abelian varieties of CM-type, Proc. Japan Acad. Ser. A Math. Sci. 82 (2006), no. 3, 25-29. [4] $\frac{}{423-440 .}$. Discrete tomography and Hodge cycles, Tohoku Math. J. (2) 59 (2007), no. 3, 